\title{
POINTWISE CONVERGENCE OF THE PRODUCT INTEGRAL FOR A CERTAIN INTEGRAL TRANSFORMATION ASSOCIATED WITH A RIEMANNIAN METRIC
}

\author{
By Yoshiaki MaEdA
}

\section{$\S 0$. Introduction.}

In [13] Inoue-Maeda present a rigorous meaning to the convergence of the path integral in a non-compact curved space. Though comparing with Feynman's original idea, they considered the case where $i \hbar^{-1}$ is replaced by $-\lambda(\lambda>0)$. Namely, they considered a certain integral transformation associated with a given Lagrangian function of the form; $L(x, \dot{x})=g_{\imath \jmath}(x) \dot{x}_{\imath} \dot{x}_{j}+V(x)$, where $G=$ $\left(g_{i j}(x)\right)$ defines a Riemannian metric and $V(x)$ is a smooth function with the compact support, and showed the convergence of its product integral in the topology of the uniform operator norm.

The purpose of this paper is to continue the above work as follows; First, we extend the above integral transformations to those which acts on sections of a general vector bundle (Cf. (0.1)). Also, we construct fundamental solutions for parabolic systems geometrically. Here, we shall deal with the case $V=0$, only for simplicity. The second aim is to show the convergence of the product integral of the integral transformation in a refined topology (pointwise convergence of the kernel function).

We suspect that these observation for the convergence of the product integral may have interesting applications, and here we can derive the asymptotic behavior of the fundamental solution for a parabolic system defined on the non-compact manifold in terms of geometrical invariants.

Let $(M, g)$ be a smooth, complete $m$-dimensional Riemannian manifold and let $E$ be a vector bundle over $M$ with a linear connection $D$. Suppose that $E$ is furnished with an inner product $\langle,\rangle_{x}$ at each fibre $E_{x}, x \in M$, preserved by $D$. Using the connection $D$, we can consider the parallel translation along the minimal geodesic $\gamma_{c}$ from $y$ to $x$, which maps an element of $E_{y}$ to that of $E_{x}$. We denote it by $P(x, y)$ (Cf. $\S 2$ ).

Denote by $C_{0}(E)$ the set of all continuous sections of $E$ with compact support and by $C^{\infty}(E)$ that of all smooth sections of $E$. Put $C_{0}^{\infty}(E)=C_{0}(E) \cap C^{\infty}(E)$.

For $\xi \in C_{0}^{\infty}(E)$, we define the $L^{2}$-norm as

Received April 2, 1984 


$$
\|\xi\|_{L^{2}(E)}=\left[\int_{M}\langle\xi(x), \xi(x)\rangle_{x} d \mu_{g}(x)\right]^{1 / 2}
$$

where $d \mu_{g}(x)$ denotes the canonical measure defined by the Riemannian metric $g$. We denote by $L^{2}(E)$ the Hilbert space of sections $\xi$ of $E$ such that $\|\xi\|_{L^{2}(E)}<+\infty$.

Now, consider the following integral transformation in $L^{2}(E)$ with parameters $0 \leqq s<t$, and $\lambda>0$ (Cf. [7], [12], [13] and [16]),

$$
\begin{aligned}
H(\lambda ; t, s) \xi(x)=\left(2 \pi \lambda^{-1}\right)^{-m / 2} \int_{M} \rho(t, s ; x, y)[ & \exp (-\lambda S(t, s ; x, y))] \\
& \times P(x, y) \xi(y) d \mu_{g}(y),
\end{aligned}
$$

for $\xi \in C_{0}^{\infty}(E)$ : Here $S(t, s ; x, y)=d^{2}(x, y) /(2(t-s))$, where $d(x, y)$ is the distance function and $\rho(t, s ; x, y)$ is defined by

$$
\rho(t, s ; x, y)=\left|\operatorname{det}\left[-\partial_{x} \partial_{y} S(t, s ; x, y)\right] / \mu_{g}(x) \mu_{g}(y)\right|^{1 / 2},
$$

where $\mu_{g}(x)=\left[\operatorname{det}\left(g_{\imath j}(x)\right)\right]^{1 / 2}((0.2)$ is assumed to be well-defined here. In fact, it is guaranteed under the assumption (A.0) which is stated later.) (Cf. [7] and [16]).

The kernel function of $H(\lambda ; t, s)$ will be denoted by $H(\lambda ; t, s ; x, y)$ which may be considered as a section on $E \otimes E^{*}$; the vector bundle over $M \times M$ whose fibre at $(x, y) \in M \times M$ is given by the tensor product $E_{x} \otimes E_{y}^{*}$.

We consider the product integral for the above operator $(0.1)$. Namely, let $\sigma_{N}$ be the $N$-equal subdivision of the interval $[0, t]$ for given $t>0$ and any positive integer $N$,

We set

$$
\sigma_{N}: 0=t_{0}<t_{1}<\cdots<t_{N-1}<t_{N}=t, \quad t_{j}=(\jmath / N) t .
$$

$$
H\left(\lambda ; \sigma_{N} \mid t\right)=H\left(\lambda ; t, t_{N-1}\right) H\left(\lambda ; t_{N-1}, t_{N-2}\right) \cdots H\left(\lambda ; t_{1}, 0\right),
$$

and denote by $H\left(\lambda ; \sigma_{N} \mid t ; x, y\right)$ the kernel function of $(0.3)$.

In order to state our results, we introduce the following assumptions :

(A.0) $(M, g)$ is a connected, simply connected, complete Riemannian manifold and has non-positive sectional curvature.

(A.1) There exists a positive constant $k_{1}$ such that for any multi-index $\alpha=\left(\alpha_{1}, \cdots, \alpha_{m}\right), 0 \leqq|\alpha| \leqq 3$ and $x \in M$,

$$
\left|\nabla^{\alpha} R_{i j k}{ }^{h}(x)\right|_{x} \leqq k_{1},
$$

where $\left.\right|_{x}$ is the norm at $x$ defined by $g$ and $\nabla$ and $R_{\imath j k}{ }^{h}$ are the Riemannian connection and the curvature tensor defined by $g$ respectively.

(A.2) There exists a positive constant $k_{2}$ such that the curvature 2 -form $\Omega$ of $D$ satisfies

$$
\left|D^{\alpha} \Omega(x)\right|_{x} \leqq k_{2} \quad \text { for } 0 \leqq|\alpha| \leqq 3 \text {, and } \quad x \in M \text {, }
$$


where ||$_{x}$ is the norm at $x$ defined by $\langle,\rangle_{x}$.

Remark. Combining the Riemannian metric $g_{i j}(x)$ and the inner product $\langle,\rangle_{x}$, we can define the norms for the sections of the tensor product bundles of the tangent bundle $T M$, the cotangent bundle $T^{*} M, E$ and $E^{*}$. Also, here we extend naturally the action of $D$ and $\nabla$ to tensor fields with values in $E$ (or $E^{*}$ ). Including these, we denote these by the same letters ||$, D$ and $\nabla$ unless there occurs no confusion.

We can state the main theorem of this paper.

MAIN THEOREM. Let $(M, g)$ be a m-dimensional Riemannian manifold and let $E$ be a vector bundle over $M$ satisfying (A.0)-(A.2). Fix $T>0$ arbitraily. Then, the limit

$$
\boldsymbol{H}(\lambda ; t ; x, y)=\lim _{N \rightarrow \infty} H\left(\lambda ; \sigma_{N} \mid t ; x, y\right)
$$

converges unformly on $M \times M$ in the norm defined by $E_{\bar{X}} E^{*}$ (Cf. §2) for any $0<t<T$. Moreover, $\boldsymbol{H}(\lambda ; t ; x, y)$ gives a fundamental solution of the following parabolic equation:

$$
\left\{\begin{array}{l}
{\left[(\partial / \partial t)-\lambda^{-1} \mathscr{H}_{x}\right] \boldsymbol{H}(\lambda ; t ; x, y)=0,} \\
\lim _{t \rightarrow 0+} \boldsymbol{H}(\lambda ; t ; x, y)=\delta_{y}(x) \otimes I d_{y}
\end{array}\right.
$$

where

$$
\mathscr{H}_{x}=(1 / 2) \Delta_{x}^{D}-(1 / 12) \operatorname{Scal}_{g}(x), \quad \Delta^{D}=-D^{*} D,
$$

$D^{*}$ is the adjoint operator of $D$ with respect to the inner product on $L^{2}(E)$ and $\operatorname{Scal}_{\mathrm{g}}(x)$ is the scalar curvature.

On the other hand, in the course of the proof of the main theorem, we can get the asymptotic behavior of $\boldsymbol{H}(\lambda ; t, s ; x, y)$ as $t \rightarrow 0+$, which is a partial extension of the results in Molchanov [15] who treated the case where $E=M \times \boldsymbol{R}$.

COROLLARY. Under the same assumptions as in the main theorem, the fundamental solution $\boldsymbol{H}(\lambda ; t, s ; x, y)$ of $(0.7)$ satısfies, for any $0<\varepsilon<1 / 2$,

$$
\begin{aligned}
& \left|\boldsymbol{H}(\lambda ; t ; x, y)-\left(2 \pi \lambda^{-1} t\right)^{-m / 2} \rho(x, y)\left[\exp \left(-\lambda\left(d^{2}(x, y) / 2 t\right)\right)\right] P(x, y)\right|_{(x, y)} \\
& \quad \leqq \boldsymbol{\gamma}^{\prime} t^{-m / 2+3 / 2}\left[\exp \left(-\lambda \varepsilon^{* *} d^{2}(x, y) / 2 t\right)\right],
\end{aligned}
$$

for any $x, y \in M$, with some positive constant $\gamma^{\prime}$, where $\varepsilon^{* *}=1-2 \varepsilon, \rho(x, y)=$ $\left|\operatorname{det}_{g}\left(d E x p_{x}^{-1}\right)_{y}\right|^{1 / 2}$ and $E_{x p}$ is the exponential mapping defined by $g$ (Cf. $\S 2$ ).

Remark. By a technical reason $\varepsilon^{* *}$ appears in the above inequality, but it seems necessary for general cases.

Acknowledgement. The author wishes to express his hearty thanks to 
Professor Atushi Inoue for his valuable suggestions and discussions. He also wishes to express his gratitude to Professors Morio Obata and Hideki Omori for their encouragement during the preparation of this paper.

\section{$\S 1$. Outline of the proof of Main theorem and related remarks.}

In this section, we state the plan to prove the main theorem in the introduction. First, in $\S 3$, we show the following basic properties of $H(\lambda ; t, s)$ defined by $(0.1)$.

Proposition 1.1. Assume (A.0)-(A.2). On fixing $T>0$ arbitrarily, the following properties hold for $0 \leqq s<t<T$ :

(a) The integral transformation $H(\lambda ; t, s)$ defines a bounded linear operator in $L^{2}(E)$.

$$
\lim _{t \rightarrow s}\|H(\lambda ; t, s) \xi-\xi\|_{L^{2}(E)}=0,
$$

Let $\mathscr{B}\left(L^{2}(E)\right.$ ) be the set of all bounded linear operators on $L^{2}(E)$ and we introduce the topology by the operator norm in it. Now, we study the convergence of the product integral of $H(\lambda ; t, 0)$ in $\mathscr{B}\left(L^{2} E\right)$ ). So, we prove the following in $\S 4-5$, which is one of the key results :

THEOREM 1.2. Under the same assumptions as in Proposition 1.1, the following properties hold:

(a) There exists a positive constant $C_{0}=C_{0}(\lambda ; T)$ such that

$$
\begin{gathered}
\left\|H\left(\lambda ; t+t^{\prime}, s\right) \xi-H\left(\lambda ; t+t^{\prime}, t^{\prime}\right) H\left(\lambda ; t^{\prime}, s\right) \xi\right\|_{L^{2}(E)} \\
\leqq C_{0}\left[\left(t+t^{\prime}-s\right)^{3 / 2}-t^{3 / 2}+\left(t^{\prime}-s\right)^{3 / 2}\right]\|\xi\|_{L^{2}(E)}
\end{gathered}
$$

for any $\xi \in L^{2}(E)$ and $0 \leqq s<t^{\prime}<t<t+t^{\prime}<T$.

(b) There exists a limit $\boldsymbol{H}(\lambda ; t)=\lim _{N \rightarrow \infty} H\left(\lambda ; t, t_{N-1}\right) \cdots H\left(\lambda ; t_{1}, 0\right), t_{j}=(j / N) t$, $\jmath=1, \cdots, N-1$, in $\mathscr{B}\left(L^{2}(E)\right)$ for any $t>0$. Therefore, $\{\boldsymbol{H}(\lambda ; t)\}_{t \geqq 0}$ with $\boldsymbol{H}(\lambda ; 0)$ =the identity operator, forms a $C^{0}$ semi-group in $L^{2}(E)$.

(c) The infinitesimal generator $\lambda^{-1} \mathscr{H}$ of $\boldsymbol{H}(\lambda ; t)$ is given by

$$
\begin{aligned}
\lambda^{-1}\left(\mathscr{H}_{x} \xi\right)(x) & =\left[\left.(\partial / \partial t) \boldsymbol{H}(\lambda ; t) \xi(x)\right|_{t=0}\right] \\
& =\lambda^{-1}\left[(1 / 2) \triangle S_{x}^{D}-(1 / 12) \operatorname{Scal}_{\boldsymbol{g}}(x)\right] \xi(x) .
\end{aligned}
$$

Theorem 1.2 shows that the product integration of (0.6) determines a fundamental solution of the heat type equation (0.8) in the distribution sense. To show the regularity, we construct a kernel function by another method (so-called Levi's method) which is rather standard in the theory of partial differential equation (Cf. Friedman [9]). Using this estimate, we prove the main theorem stated in $\S 0$. Namely, we show in $\S 7$ the following: 
THEOREM 1.3. Under the same assumptions as in Proposition 1.1, we can construct a fundamental solution $\boldsymbol{H}(\lambda ; t)$ with the following estimate: For any $0<\varepsilon<1 / 4$, there exists a positive constant $\gamma=\gamma(\lambda ; T, \varepsilon)$ which dose not depend on $\sigma_{N}$ such that

$$
\begin{aligned}
& \left|\boldsymbol{H}(\lambda ; t ; x, y)-H\left(\lambda ; \sigma_{N} \mid t ; x, y\right)\right|_{(x, y)} \\
& \left.\quad \leqq \gamma t^{-(m-3) / 2} N^{-1 / 2}\left[\exp \left(-\lambda \varepsilon^{(4)} d^{2}(x, y) / 2 t\right)\right)\right]
\end{aligned}
$$

where $\varepsilon^{(4)}=1-4 \varepsilon$ and $\boldsymbol{H}(\lambda ; t ; x, y)$ is the kernel function of $\boldsymbol{H}(\lambda ; t)$.

Remark 1. We cannot prove the convergence of $H\left(\lambda ; \sigma_{N} \mid t ; x, y\right)$ without constructing the fundamental solution by Levi's method. This may be still an interesting problem.

For the sake of our computations, we shall introduce the local coordinate expression. Given $\bar{x} \in M$, let $U$ be a local coordinate neighborhood of $\bar{x}$ with the coordinate $\left(x^{1}, \cdots, x^{m}\right)$ such that $\left.E\right|_{U}$ is trivialized as $\left.E\right|_{U} \cong U \times \boldsymbol{F}$, where $\boldsymbol{F}$ is the standard fibre of $E$. Taking a frame field $\left\{e_{a}(x)\right\}$ of $\left.E\right|_{U}$ (i.e. $e_{a}(x)$ depends smoothly on $x \in U$ and $\left\{e_{a}(x)\right\}$ forms a basis on $\boldsymbol{F}$ for any $\left.x \in U\right)$.

Denote by $\Gamma_{j b}^{a}(x)$ the component of $D_{j}=D_{\left(\partial / \partial x^{\prime}\right)}$. Then, for each $\xi \in C^{\infty}(E)$, its covariant derivative $D$, can be expressed by

$$
\left(D_{j} \xi\right)^{a}(x)=\partial_{j} \xi^{a}(x)+\Gamma_{j b}^{a}(x) \xi^{b}(x) .
$$

Also, for any $\phi \in \Omega^{1}(E)$, a $E$-valued 1-form, expressed by $\psi(x)=\psi_{i}(x) d x^{2}$ with $\phi_{i}(x)=\phi_{i}{ }^{a}(x) e_{a}(x)$, we have

$$
\left(D_{j} \psi\right)_{i}^{a}(x)=\partial_{\jmath} \phi_{i}^{a}(x)-\left\{\begin{array}{l}
k \\
j i
\end{array}\right\}(x) \phi_{k}^{a}(x)+\Gamma_{j b}^{a}(x) \psi_{\imath}^{b}(x),
$$

where $\left\{\begin{array}{c}k \\ j i\end{array}\right\}(x)$ is the Christoffel symbol of $g$. Moreover, the local coordinate expression of the covariant derivatives for any tensor field with values in $E$ is obtained similarly. Using these notations, $\Delta^{D}$ can be expressed as

$$
\left(\Delta^{D} \xi\right)^{a}(x)=g^{\imath \jmath}(x)\left[\delta_{b}^{a} \partial_{i}+\Gamma_{\imath b}^{a}(x)\right]\left[\delta_{c}^{b} \partial_{j}+\Gamma_{j c}^{b}(x)\right] \xi^{c}(x),
$$

for any $\xi \in C^{\infty}(E)$.

Finally, we give some remarks about Main Theorem.

Remark 2. (i) Trivial bundle, $E=M \times \boldsymbol{R}$ (or $M \times \boldsymbol{C}$ ). A section of the trivial bundle can be identified with a function on $M$ and $C(E) \cong C(M)$. Taking the trivial connection, i.e. $P(x, y)=i d$., we get a integral transformation acting for functions on $M$, which is considered in [12]. So, in this case the limit

$$
\lim _{N \rightarrow \infty} \int_{M} \cdots \int_{M} H\left(\lambda ; t, t_{N-1} ; x, z_{N-1}\right) \cdots H\left(\lambda ; t_{1}, 0 ; z_{1}, y\right) d \mu_{g}\left(z_{N-1}\right) \cdots d \mu_{g}\left(z_{1}\right)
$$

exists as a function on $M \times M$ for fixed $t, 0<t<T$, under the assumptions (A.0)- 
(A.1). We may denote its limit by

$$
\int_{C_{t, 0 ; x, y}}[\exp (-\lambda S(\gamma))] \mathscr{D}_{F}(\gamma) \quad \text { (Cf. Feynman }[8] \text { ), }
$$

where $\mathcal{C}_{t, 0 ; x, y}$ is the path space from $y$ at $t=0$ to $x$ at $t=t, S(\gamma)$ is the classical action along the path $\mathcal{C}_{t, 0 ; x, y}$ and $\mathscr{D}_{F}(\gamma)$ is the 'notorious' Feynman measure on $\mathcal{C}_{t, 0 ; x, y}$.

(ii) The bundle of $p$-forms, $E=\Lambda^{p} T^{*} M$. In this bundle, we can induce the inner product $\langle,\rangle_{x}$ and the connection $D$ cannonically by $g$. Namely, for $\xi(x)$ $=\xi_{i_{1} \cdots \imath} p(x) d x^{\imath_{1}} \wedge \cdots \wedge d x^{\imath} p, \quad \eta(x)=\eta_{i_{1} \cdots \imath}(x) d x^{\imath_{1}} \wedge \cdots \wedge d x^{\imath} p \in C(E)$, we define the inner product and the covariant derivative by

$$
\langle\xi(x), \eta(x)\rangle_{x}=g^{\imath_{1} \jmath_{1}}(x) \cdots g^{\imath^{\jmath} p} p(x) \xi_{\imath_{1} \cdots \imath_{p}}(x) \eta_{\jmath_{1} \cdots j_{p}}(x),
$$

and

$$
\left(D_{j} \xi\right)_{i_{1} \cdots \imath_{p}}(x)=\partial_{j} \xi_{\imath_{1} \cdots \imath_{p}}(x)-\left\{\begin{array}{c}
k \\
j i_{1}
\end{array}\right\}(x) \xi_{k \imath_{2}} \cdots_{i_{p}}(x)-\cdots-\left\{\begin{array}{c}
k \\
j i_{p}
\end{array}\right\}(x) \xi_{\imath_{1} \cdots \imath_{p-1} k}(x) .
$$

Then, we get the operator $\mathscr{H}_{x}=-(1 / 2) \Delta_{L}-(1 / 12) \operatorname{Scal}_{g}(x)$ : Here $\Delta_{L}$ is the rough Laplacian defined by $g$ (Cf. [14]), and it is given by

$$
\begin{aligned}
& \left(\Delta_{L} \xi\right)_{i_{1} \cdots \imath_{p}}(x)=\left(\Delta_{H} \xi\right)_{i_{1} \cdots \nu_{p}}(x)+\sum_{r=1}^{p} R_{\imath_{r}}{ }^{\jmath}(x) \xi_{\imath_{1} \cdots \cdots \imath_{p}}(x) \\
& +\sum_{r>s} R_{\imath_{r} \imath_{s}}^{j k}(x) \xi_{\imath_{1} \cdots \cdots k \cdots \cdots \imath_{p}}(x),
\end{aligned}
$$

where $R_{\imath \jmath}(x)$ and $R_{\imath j k}{ }^{h}(x)$ are the component of the Ricci tensor and the curvature tensor of $g$ respectively, and $\Delta_{H}$ denotes the Hodge-de Rham operator.

(iii) As a generalization of (ii), one may construct the fundamental solution of the parabolic equation whose infinitesimal generator is the following:

(a) The Lichnerowicz Laplacian acting on tensor fields.

(b) The spinorial Laplacian of Lichnerowicz when $M$ admits a spinorial structure.

\section{$\S 2$. Classical action and parallel translation.}

Throughout this paper, notations and definitions concerning the differential geometry will be refered to [3] and [13].

We recall a geodesic, i. e. a curve $\gamma(\tau)$ which satisfies the following differential equation,

$$
\frac{\delta^{2} \gamma^{2}(\tau)}{\delta \tau^{2}} \equiv \frac{d^{2} \gamma^{2}(\tau)}{d \tau^{2}}+\left\{\begin{array}{c}
2 \\
\jmath k
\end{array}\right\}(\gamma(\tau)) \frac{d \gamma^{3}(\tau)}{d \tau} \cdot \frac{d \gamma^{k}(\tau)}{d \tau}=0
$$

where $\delta / \delta \tau$ denotes the covariant derivative along the curve $\gamma(\tau)$. Given $x \in M$, we define a mapping $\operatorname{Exp}_{x}$ from the tangent space $T_{x} M$ into $M$ by $\operatorname{Exp}_{x} \tau X=$ $\gamma(\tau)$, where $\gamma(\tau)$ satisfies $(2.1)$ with the initial conditions $\gamma(0)=x, \dot{\gamma}(0)=X \in T_{x} M$. 
By the assumption (A.0), Exp $p_{x}$ gives a diffeomorphism from $T_{x} M$ onto $M$.

For each $X \in T_{x} M$, identifying $T_{X}\left(T_{x} M\right)$ with $T_{x} M$, we may induce naturally the scalar product in $T_{X}\left(T_{x} M\right)$. We denote by $\left(d E x p_{x}\right)_{X}$ the differential mapping of $\operatorname{Exp}_{x}$ at $X$. Define also the function $\theta(x, y)$ on $M \times M$ by $\theta(x, y)=$ $\left|\operatorname{det}_{g}\left(d E x p_{x}\right)_{X}\right|$ (Cf. [3]). Then, the function $\rho(t, s ; x, y)$ defined by $(0.2)$ can be written as

$$
\rho(t, s ; x, y)=(t-s)^{-m / 2} \rho(x, y),
$$

where $\rho(x, y)=\theta(x, y)^{-1 / 2}$.

To give the estimate of $\rho(x, y)$, recall a Jacobi field $J(\tau)$ along the geodesic $\gamma(\tau)$. By (A.0)-(A.1) and the Rauch comparison theorem we get the following (Cf. [4]): Let $J(\tau)$ be a Jacobi field along geodesic $\gamma(\tau)=E x p_{x} \tau \omega,|\omega|=1$ with initial conditions $J(0)=0, \dot{J}(0) \neq 0$. Then, there exists a positive constant $k_{3}$ independent of $x$ such that for any $y \in M$,

$$
r|\dot{J}(0)|_{x} \leqq|J(r)|_{y} \leqq\left(\sinh k_{3} r / k_{3} r\right)|\dot{J}(0)|_{x}
$$

where $r=d(x, y)$. In particular, we have

$$
|\rho(x, y)| \leqq 1 .
$$

Denote by $S M$ and $S_{x} M$ the unit sphere bundle over $M$ and the fibre of $S M$ at $x \in M$ respectively. Using the Jacobi equation and (A.1), we have

LEMma 2.1. Assume that (A.0)-(A.1) hold. Given any $x, y \in M$, there exists a positive constant $k_{4}$ such that

$$
|\dot{J}(r)|_{y} \leqq\left(k_{4} \exp \left(k_{4} r\right)\right)|\dot{J}(0)|_{x} .
$$

Moreover, we have

$$
\left|\rho_{r}(x, y)\right| \leqq k_{4} \exp \left(k_{4} r\right), \quad r=d(x, y),
$$

where $\rho_{r}(x, y)=(d / d r) \rho\left(x, \operatorname{Exp}_{x} r \omega\right), \operatorname{Exp} p_{x} r \omega=y, \omega \in S_{x} M$.

Now, we give an estimate of the higher order derivatives of the functions $\rho(x, y)$, which will be proved in Appendix.

Proposition 2.2. Assume that $(M, g)$ satısfies (A.0)-(A.1). Then, there exists $a$ positive constant $k_{5}$ such that for any $x, y \in M$,

$$
\left|\nabla_{y}^{\alpha} \rho(x, y)\right| \leqq k_{5} \exp \left(k_{5} r\right), \quad r=d(x, y), \quad 0 \leqq|\alpha| \leqq 3 .
$$

Next, we recall the parallel transformation of a section of the vector bundle $E$ by the connection $D$. Given a curve $\gamma(\tau)$ on $M$ such that $\gamma(s)=y, \gamma(t)=x$, $s<t$, and $\xi(y) \in E_{y}$, define $\tilde{\xi}(\tau) \in E_{\gamma(\tau)}$ by 


$$
\frac{\delta}{\delta \tau} \tilde{\xi}(\tau)=D_{\dot{\gamma}(\tau)} \tilde{\xi}(\tau)=0, \quad \tilde{\xi}(0)=\xi(y) .
$$

We write $\tilde{\xi}(t)$ by $P_{s}^{t}(D, \gamma) \xi(y)$. Since (2.8) is a first order differential equation, the solution of (2.8) exists uniquely for any given curve $\gamma(\tau)$. In particular, if $\gamma_{c}(\tau)$ be a classical path which satisfies $(2.1)$ with $\gamma_{c}(0)=y, \gamma_{c}(t)=x$, then we have

$$
P_{s}^{t}\left(D, \gamma_{c}\right) \xi(y)=P_{0}^{1}\left(D, \tilde{\gamma}_{c}\right) \xi(y),
$$

where $\tilde{\gamma}_{c}(\tilde{\tau})=\gamma_{c}(s+\tilde{\tau}(t-s))$, for any $x \in M$ and any $y \in M$. Moreover, we write $P_{0}^{1}\left(D, \tilde{\gamma}_{c}\right)$ by $P(x, y)$ for simplicity.

Since each vector spaces $E_{x}$ and $E_{y}^{*}$ are equipped with the inner products, we can induce the inner product on $E_{x} \otimes E_{y}^{*}$, which will be denoted by $\langle,\rangle_{(x, y)}$. That is, given $\xi(x, y)=\xi(x) \nabla \xi^{*}(y), \eta(x, y)=\eta(x) \otimes \eta^{*}(y)$, we put

$$
\langle\xi(x, y), \eta(x, y)\rangle_{(x, y)}=\langle\xi(x), \eta(x)\rangle_{x} \cdot\left\langle\xi^{*}(y), \eta^{*}(y)\right\rangle_{y} .
$$

Also, we denote the norm on $E_{x} \otimes E_{y}^{*}$ by

$$
|\xi(x, y)|_{(x, y)}=\langle\xi(x, y), \xi(x, y)\rangle_{(x, y)}^{1 / 2} .
$$

We extend $\nabla$ and $D$ to a tensor fields with the values in $E$, and denote also them by the same letter.

The following is obvious from the definition (2.8) (Cf. [2]):

Lemma 2.3. For any $x, y \in M$, we get

(i) $P(x, y)$ is a smooth (local) section on $E \otimes E^{*}$.

(ii) $P(x, x)=I d$., the identity operator on $E_{x}$.

(iii) $\left\langle\nabla_{x} d^{2}(x, y), D_{x} P(x, Y)\right\rangle_{x}=0$.

Lemma 2.4. Assume (A.0)-(A.2). Given $\xi \in C^{\infty}(E)$, we get

$$
\|P(x, y) \xi(y)\|_{x}=\|\xi(y)\|_{y},
$$

for any $x, y \in M$.

Proof. Consider (2.8). Then, we get

$$
\begin{aligned}
\frac{d}{d \tau}\langle\tilde{\xi}(\tau), \hat{\xi}(\tau)\rangle_{\gamma(\tau)} & =2\left\langle D_{\dot{\gamma}(\tau)} \bar{\xi}(\tau), \bar{\xi}(\tau)\right\rangle_{\gamma(\tau)} \\
& =0 .
\end{aligned}
$$

Using (2.12) and (A.2), we get (2.11).

The following properties are useful throughout our computations:

LEMMA 2.5. $P(x, y)$ satısfies the following.

$$
D_{x} P(x, y)_{1 x=y}=0, \quad \Delta_{x}^{D} P(x, y)_{\mid x=y}=0 .
$$




$$
D_{y} P(x, y)_{1 y=x}=0, \quad \Delta_{y}^{D} P(x, y)_{1 y=x}=0 .
$$

Proof. Let $\left\{e_{a}(y)\right\}_{a=1}^{p}$ be an orthonormal basis at $E_{y}$, where $\operatorname{dim} E_{y}=p$. Extend $\left\{e_{a}\right\}$ to a local frame field so that they are parallel. Take a normal coorinate $\left(y^{1}, \cdots, y^{m}\right)$ at $y$ and denote by $\Gamma_{\jmath \alpha}^{b}$ the coefficients of $D$. By putting $\hat{\xi}(\tau)=\tilde{\xi}^{a}(\tau) e_{a}\left(\gamma_{c}(\tau)\right)$, (2.8) can be written as

$$
\frac{d \tilde{\xi}^{a}(\tau)}{d \tau}+\Gamma_{j b}^{a}\left(\gamma_{c}(\tau)\right) \frac{d \gamma^{\jmath}(\tau)}{d \tau} \tilde{\xi}^{b}(\tau)=0
$$

So, using the Taylor expansion, we can write $\tilde{\xi}(\tau)$ by

$$
\tilde{\xi}^{a}(\tau)=\hat{\xi}^{a}(0)+\left(\tilde{\xi}^{a}\right)^{\prime}(0) \tau+(1 / 2)\left(\tilde{\xi}^{a}\right)^{\prime \prime}(0) \tau^{2}+O\left(\tau^{3}\right),
$$

where $\left(\tilde{\xi}^{a}\right)^{\prime}(0)=(d / d \tau) \tilde{\xi}^{a}(0)$, etc. Differentiating (2.15) successively with respect to $\tau$, we get

$$
\begin{gathered}
\left(\tilde{\xi}^{a}\right)^{\prime}(0)=-\Gamma_{j b}^{a}(y) Y^{\jmath} \xi^{b}(y) \\
\left(\tilde{\xi}^{a}\right)^{\prime \prime}(0)=-\left[\partial_{l} \Gamma_{j b}^{a}(y) Y^{l} Y^{\jmath}+\Gamma_{\jmath c}^{a}(y) \Gamma_{l b}^{c}(y) Y^{j} Y^{l}\right] \xi^{b}(y),
\end{gathered}
$$

where $\operatorname{Exp}_{y} Y=x$, because $\left\{\begin{array}{c}\imath \\ j k\end{array}\right\}(y)=0$. Substituting (2.17) and (2.18) into (2.16) and putting $\tau=1$, we have

$$
\begin{aligned}
\tilde{\xi}^{a}(1)= & P_{b}^{a}(x, y) \xi^{b}(y) \\
= & {\left[\delta_{b}^{a}-\Gamma_{j b}^{a}(y) Y^{\jmath}-(1 / 2)\left[\partial_{l} \Gamma_{\jmath b}^{a}(y) Y Y^{\jmath}\right.\right.} \\
& \left.\left.\quad+\Gamma_{\jmath c}^{a}(y) \Gamma_{l b}^{c}(y) Y Y^{j}\right]+O\left(Y^{3}\right)\right] \xi^{b}(y),
\end{aligned}
$$

where $P_{b}^{a}(x, y)$ is the component of $P(x, y)$ with respect to $\left\{e_{a}\right\}$. Recall

$$
\begin{aligned}
D_{x, k} D_{x, j} P_{b}^{a}(x, y)= & \partial_{k} \partial_{j} P_{b}^{a}(x, y)+\partial_{k} \Gamma_{j c}^{a}(Y) P_{b}^{c}(x, y) \\
& +\Gamma_{j c}^{a}(Y) \partial_{k} P_{b}^{c}(x, y)+\Gamma_{k c}^{a}(Y) \partial_{j} P_{b}^{c}(x, y) \\
= & -(1 / 2)\left[\partial_{k} \Gamma_{j b}^{a}(y)+\partial_{j} \Gamma_{k b}^{a}(y)+2 \Gamma_{k c}^{a}(y) \Gamma_{j b}^{c}(y)\right] \\
& +\partial_{k} \Gamma_{j b}^{a}(y)+\Gamma_{k c}^{a}(y) \Gamma_{j b}^{a}(y)+O(Y),
\end{aligned}
$$

which proves the second equality of (2.13). For (2.14), remark that for any $x, z \in M$, we have

$$
P(x, z) P(z, x)=P(x, x) \text {. }
$$

Differentiating (2.22) covariantly and using Lemma 2.3 (ii) and (2.13), we obtain (2.14).

Lastly, we get an estimate for the higher order derivatives of $P(x, y)$, which will be shown in Appendix. 
Proposition 2.6. Assume that $(M, g)$ satısfies (A.0)-(A.2). Then, there exists $a$ positive constant $k_{4}^{\prime}$ such that for any $x, y \in M$ and for $0 \leqq|\alpha| \leqq 3$,

$$
\left\{\begin{array}{l}
\left|\left(D_{x}\right)^{\alpha} P(x, y)\right|_{(x, y)} \leqq k_{4}^{\prime} \exp \left(k_{4}^{\prime} r\right), \\
\left|\left(D_{y}\right)^{\alpha} P(x, y)\right|_{(x, y)} \leqq k_{4}^{\prime} \exp \left(k_{4}^{\prime} r\right), \quad r=d(x, y) .
\end{array}\right.
$$

\section{$\S 3$. Basic properties of $H(\lambda ; t, s)$.}

Recall the operator $H(\lambda ; t, s)$ in $(0.1)$. Using the notations as in $\S 2$, it can be written as follows :

$$
\begin{array}{r}
H(\lambda ; t, s) \xi(x)=\left(2 \pi \lambda^{-1}\right)^{-m / 2} \int_{M} \rho(t, s ; x, y)\left[\exp \left(-\lambda\left(d^{2}(x, y) / 2(t-s)\right)\right)\right] \\
\times P(x, y) \xi(y) d \mu_{\boldsymbol{g}}(y)
\end{array}
$$

for $\xi \in C_{0}(E)$.

In this section, we shall give some properties of (3.1). Using (2.4) and (2.11) and copying the proof of Lemma 2.1 [13], we get the following, which implies the part of (a) in Proposition 1.1:

Proposition 3.1. Assume that $(M, g)$ satisfies (A.0)-(A.2) and fix $T>0$ arbitrarily. Then, the operator $H(\lambda ; t, s)$ is stable, that is, there exists a positive constants $C_{1}=C_{1}(\lambda ; T)$ such that

$$
\|H(\lambda ; t, s) \xi(x)\|_{L^{2}(E)} \leqq\left(\exp \left(C_{1}(t-s)\right)\right)\|\xi\|_{L^{2}(E)}
$$

for $0 \leqq s<t<T$ and $\xi \in C_{0}^{\infty}(E)$.

Next, we study the behavior of $H(\lambda ; t, s)$ as $t \downarrow s$. Namely, we have the following which is the part (b) in Proposition 1.1:

Proposition 3.2. Under the same assumptions as in Proposition 3.1, we have for any $0 \leqq s<t<T$,

$$
\lim _{t \downarrow s}\|H(\lambda ; t, s) \xi-\xi\|_{L^{2}(E)}=0,
$$

for any $\xi \in C_{0}^{\infty}(E)$. Therefore, for fixed $s \geqq 0$, putting $H(\lambda ; s, s)=$ the identity transformation, we have the mapping from $t \in[s, T)$ to $H(\lambda ; t, s) \in L^{2}(E)$, strongly continuous in $t$ for each $\xi \in L^{2}(E)$. Also, the similar statement in $s$ as above holds.

Proof. By Proposition 3.1, it is sufficient to prove (3.3) for each $\xi \in C_{0}^{\infty}(E)$. We define a cut off function $\chi \in C_{0}^{\infty}(M)$ as $\chi(x) \equiv 1$ if $d(x$, supp $\xi) \leqq 2$ and $\equiv 0$ if $d(x, \operatorname{supp} \xi) \geqq 3$. We show the following :

$$
\lim _{t \downarrow s}\left\|H_{1}(\lambda ; t, s) \xi-\xi\right\|_{L^{2}(E)}=0,
$$




$$
\lim _{t \downarrow s}\left\|H_{2}(\lambda ; t, s) \xi\right\|_{L^{2}(E)}=0,
$$

where $H_{1}(\lambda ; t, s)=\chi(x) H(\lambda ; t, s) \xi(x)$ and $H_{2}(\lambda ; t, s)=(1-\chi(x) H(\lambda ; t, s) \xi(x)$.

For proving (3.4), putting $y=E x p_{x} r \omega, \omega \in S_{x} M$, we get

$$
\tilde{\xi}(x, y)=\xi(x)+\tilde{\xi}_{1}(x ; r \omega), \quad \rho(x, y)=1+\rho_{1}(x ; r \omega),
$$

where $\tilde{\xi}(x, y)=P(x, y) \xi(y) \in E_{x}$ and

$$
\begin{aligned}
& \tilde{\xi}_{1}(x ; r \omega)=\int_{0}^{r} D_{\gamma_{c}(\tau)} \tilde{\xi}\left(x, \gamma_{c}(\tau)\right) d \tau \\
& \rho_{1}(x ; r \omega)=\int_{0}^{r}(d / d \tau) \rho\left(x, \gamma_{c}(\tau)\right) d \tau,
\end{aligned}
$$

where $\gamma_{c}(\tau)=E x p_{x} \tau \omega$. Using Lemma 2.1 and Proposition 2.6, we get the following, which is similar to that in Proposition 2.2, [13]:

$$
\begin{aligned}
\left|H_{1}(\lambda ; t, s) \xi(x)-\xi(x)\right|_{x} \leqq & C_{2} \chi(x) \operatorname{vol}\left(S^{m-1}\right)(t-s)^{1 / 2} \sup _{x \in M}\left[|D \xi|_{x}+|\xi|_{x}\right] \\
& \times \int_{0}^{\infty} r^{m} \exp \left(-\left[\lambda r^{2} / 2(t-s)-k_{8}(t-s) r\right]\right) d r
\end{aligned}
$$

with some positive constants $C_{2}$ and $k_{8}$. Therefore, by integrating (3.6), there exists a positive constant $C_{2}^{\prime}=C_{2}^{\prime}(\lambda ; T)$ depending on the support of $\xi$ such that

$$
\left\|H_{1}(\lambda ; t, s) \xi-\xi\right\|_{L^{2}(E)} \leqq C_{2}^{\prime}(t-s)^{1 / 2} \sup _{x \in M}\left[|D \xi|_{x}+|\xi|_{x}\right]
$$

which gives (3.4).

Also, following the same way as in Proposition 2.2, [13] and using Lemma 2.1, Proposition 2.2 and Proposition 2.6, we get (3.5).

For later use, we give some properties of the kernel function $H(\lambda ; t, s ; x, y)$ of (3.1), which is proved analogously as in Proposition 3.2.

LEMma 3.3. Assume (A.0)-(A.2). Let $\xi(\tau, y)$ be a continuous, bounded mapping from $[s, t] \times M$ to $E$ such that for any fixed $\tau, \xi(\tau, \cdot) \in C(E)$, and put

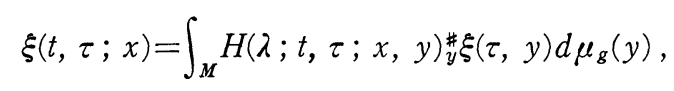

where $0 \leqq s<\tau<t<T$, and ${ }_{y}^{\#}$ is the inner product between $E_{y}$ and $E_{y}^{*}$. Then, the following properties hold:

$$
\begin{aligned}
& (\partial / \partial t) \xi(t, \tau ; x)=\int_{M}(\partial / \partial t) H(\lambda ; t, \tau ; x, y) \# \xi(\tau, y) d \mu_{g}(y), \\
& \left(D_{x}\right)^{\alpha} \xi(t, \tau ; x)=\int_{M}\left(D_{x}\right)^{\alpha} H(\lambda ; t, \tau ; x, y)_{y}^{\#} \xi(\tau, y) d \mu_{g}(y), \\
& 0 \leqq|\alpha| \leqq 2,
\end{aligned}
$$

and 


$$
\lim _{\substack{t>\tau \geq s, t \rightarrow s}} \xi(t, \tau ; x)=\xi(s, x)
$$

Similarly, let $\xi^{*}(\tau, y)$ be a continuous, bounded mapping from $[s, t] \times M$ to $E^{*}$ such that for each fixed $\tau, \xi^{*}(\tau, \cdot) \in C\left(E^{*}\right)$. We get

LEMMA 3.4. Under the same assumptions as in Lemma 3.3, we have

$$
\lim _{\substack{s \leq \tau<t \\ t \rightarrow s}} \int_{M} \xi^{*}(\tau, x)_{x}^{\#} H(\lambda ; t, s ; x, y) d \mu_{g}(x)=\xi^{*}(t, x) \text {. }
$$

\section{$\S 4$. Convergence of the product integral in the operator norm.}

In this section we shall show the parts (a) and (b) in Theorem 1.2. Take $T>0$ arbitrarily and fix it.

By the direct computation using the Hamilton-Jacobi equation for $S(t, s ; x, y)$, the continuity equation for $\rho(t, s ; x, y)$ (Cf. Lemma 1.1 and Lemma 1.5 in [13]), and Lemma 2.3 (iii), we have the following properties for the kernel function $H(\lambda ; t, s ; x, y)$ :

$$
\begin{aligned}
& {\left[(\partial / \partial s)+(2 \lambda)^{-1} \Delta_{y}^{D}\right] H(\lambda ; t, s ; x, y)} \\
& \quad=(2 \lambda)^{-1}\left(2 \pi \lambda^{-1}\right)^{-m / 2} e^{-\lambda s}\left[2\left\langle\nabla_{y} \rho, D_{y} P\right\rangle+\Delta_{y} \rho P+\rho \Delta_{y}^{D} P\right]
\end{aligned}
$$

and

$$
\begin{aligned}
& {\left[(\partial / \partial t)-(2 \lambda)^{-1} \Delta_{x}^{D}\right] H(\lambda ; t, s ; x, y)} \\
& \quad=-(2 \lambda)^{-1}\left(2 \pi \lambda^{-1}\right)^{-m / 2} e^{-\lambda s}\left[2\left\langle\nabla_{x} \rho, D_{x} P\right\rangle+\Delta_{x} \rho P+\rho \Delta_{x}^{D} P\right] .
\end{aligned}
$$

For $\xi \in C_{0}^{\infty}(E)$ and $0 \leqq s<t<t^{\prime}<T$, we may write

$$
\begin{aligned}
& H\left(\lambda ; t+t^{\prime}, s\right) \xi(x)-H\left(\lambda ; t+t^{\prime}, t^{\prime}\right) H\left(\lambda ; t^{\prime}, s\right) \xi(x) \\
& \quad=\int_{M} H\left(\lambda ; t, t^{\prime}, s ; x, y\right)_{y}^{\# \xi(y) d \mu_{g}(y),}
\end{aligned}
$$

where

$$
\begin{aligned}
& H\left(\lambda ; t, t^{\prime}, s ; x, y\right) \\
& \quad=H\left(\lambda ; t+t^{\prime}, s ; x, y\right)-\int_{M} H\left(\lambda ; t+t^{\prime}, t^{\prime} ; x, z\right)_{z}^{\#} H\left(\lambda ; t^{\prime} x ; z, y\right) d \mu_{g}(z) .
\end{aligned}
$$

Since $H(\lambda ; t, s ; x, y)$ has a singularity at $t=s$, we define, for positive $\varepsilon$,

$$
\begin{aligned}
& H^{\varepsilon}\left(\lambda ; t, t^{\prime}, s ; x, y\right) \\
& \quad=\int_{s+\varepsilon}^{t} \frac{d}{d \sigma} \int_{M} H\left(\lambda ; t+t^{\prime}, \sigma ; x, z\right)_{z}^{\#} H(\lambda ; \sigma, s ; z, y) d \mu_{g}(z),
\end{aligned}
$$

which satisfies $\lim _{\varepsilon \downarrow 0} H^{\varepsilon}\left(\lambda ; t, t^{\prime}, s ; x, y\right)=H\left(\lambda ; t, t^{\prime}, s ; x, y\right)$ for any $\left(t, t^{\prime}, s, x, y\right)$, $x \neq y$. Exchanging the differentiation and the integral in (4.4), we have 


$$
\begin{aligned}
& H^{\varepsilon}\left(\lambda ; t, t^{\prime}, s ; x, y\right) \\
& =-\int_{s+\varepsilon}^{t}\left[2 \pi \lambda^{-1}\left(t+t^{\prime}-\sigma\right)\right]^{-m / 2}\left[2 \pi \lambda^{-1}(\sigma-s)\right]^{-m / 2}(2 \lambda)^{-1} \\
& \quad \quad \times \int_{M} \sum_{i=1}^{3} h_{i}\left(\lambda ; t, t^{\prime}, s, \sigma ; x, y, z\right) d \mu_{g}(z) d \sigma,
\end{aligned}
$$

where

$$
\begin{aligned}
& h_{1}\left(\lambda ; t, t^{\prime}, s, \sigma ; x, y, z\right) \\
& =\left[\Delta_{z} \rho(x, z)-\Delta_{z} \rho(y, z)\right]\left[\exp \left(-\lambda\left[S\left(t+t^{\prime}, \sigma ; x, z\right)+S(\sigma, x ; z, y)\right]\right)\right] \\
& \quad \times P(x, z)_{z}^{\#} P(z, y), \\
& h_{2}\left(\lambda ; t, t^{\prime}, s ; x, y, z\right) \\
& =\left[\exp \left(-\lambda\left[S\left(t+t^{\prime}, \sigma ; x, z\right)+S(\sigma, s ; z, y)\right]\right)\right] \\
& \quad \times\left[\left\langle\nabla_{z} \rho(x, z), D_{z} P(x, z)\right\rangle_{z z}^{\#} P(z, y)-P(x, z)_{z}^{\#}\left\langle\nabla_{z}(y, z), D_{z} P(y, z)\right\rangle_{z}\right]
\end{aligned}
$$

and

$$
\begin{aligned}
& h_{3}\left(\lambda ; t, t^{\prime}, s, \sigma ; x, y, z\right) \\
&= {\left[\exp \left(-\lambda\left[S\left(t+t^{\prime}, \sigma ; x, z\right)+S(\sigma, s ; z, y)\right]\right)\right] \rho(x, z) \rho(z, y) } \\
& \times\left[\Delta_{z} P(x, z)_{z}^{\#} P(z, y)-P(x, z)_{z}^{\#} \Delta_{z} P(z, y)\right] .
\end{aligned}
$$

Consider each terms $h_{i}\left(\lambda ; t, t^{\prime}, s, \sigma ; x, y, z\right), i=1,2,3$, in (4.6-8). Remark that there exists a positive constant $k_{8}$ such that

and

$$
\begin{aligned}
\left|\Delta_{z} \rho(x, z)-\Delta_{z} \rho(x, z)_{\mid z=x}\right| & \leqq \int_{0}^{r}\left|\nabla_{\dot{\gamma}_{c}(\tau)} \Delta_{z} \rho\left(x, \gamma_{c}(\tau)\right)\right| d \tau \\
& \leqq k_{8} d(x, z) \exp \left(k_{8} d(x, z)\right)
\end{aligned}
$$

$$
\left|\Delta_{z} \rho(x, z)_{\mid z=x}-\Delta_{z} \rho(y, z)_{\mid z=y}\right| \leqq k_{8} d(x, y) \exp \left(k_{8} d(x, y)\right)
$$

because of Proposition 2.2. By (4.9), (4.10) and using Proposition 2.6, we have with some constant $k_{9}>0$,

$$
\begin{aligned}
& \left|h_{1}\left(\lambda ; t, t^{\prime}, s, \sigma ; x, y, z\right)\right|_{(x, y)} \\
& \quad \leqq 2 k_{9}\left[d(x, z) \exp \left(k_{8} d(x, z)\right)+d(y, z) \exp \left(k_{8} d(y, z)\right)\right] \\
& \quad \times \exp \left(-\lambda\left[S\left(t+t^{\prime}, \sigma ; x, z\right)+S(\sigma, s ; z, y)\right]\right) .
\end{aligned}
$$

By a similar computation as in [13] using Proposition 2.2 and 2.6, there exists a constant $C_{3}=C_{3}(\lambda ; T)>0$ such that for any $i=1,2,3$,

$$
\begin{aligned}
\int_{M} \int_{M}\left[2 \pi \lambda^{-1}\left(t+t^{\prime}-\sigma\right)\right]^{-m / 2}\left[2 \pi \lambda^{-1}(\sigma-s)\right]^{-m / 2} \\
\quad \times\left|h_{i}\left(\lambda ; t, t^{\prime}, s, \sigma ; x, y, z\right)\right|_{(x, y)} d \mu_{g}(z) d \mu_{g}(y) \\
\leqq C_{3}\left[\left(t+t^{\prime}-\sigma\right)^{1 / 2}+(\sigma-s)^{1 / 2}\right],
\end{aligned}
$$


and

$$
\begin{aligned}
& \int_{M} \int_{M}\left[2 \pi \lambda^{-1}\left(t+t^{\prime}-\sigma\right)\right]^{-m / 2}\left[2 \pi \lambda^{-1}(\sigma-s)\right]^{-m / 2} \\
& \quad \times\left|h_{i}\left(\lambda ; t, t^{\prime}, s, \sigma ; x, y, z\right)\right|_{(x, y)} d \mu_{g}(z) d \mu_{g}(x) \\
& \leqq C_{3}\left[\left(t+t^{\prime}-\sigma\right)^{1 / 2}+(\sigma-s)^{1 / 2}\right] .
\end{aligned}
$$

Taking a limit as $\varepsilon \rightarrow 0+$, and doing the similar computations as in [13], §3, we have

Proposition 4.1. For any $t, t^{\prime}, s, 0 \leqq s<t^{\prime}<t<t+t^{\prime}<T$ and $\xi \in L^{2}(E)$, the following inequality holds:

$$
\begin{gathered}
\left\|H\left(\lambda ; t+t^{\prime}, s\right) \xi-H\left(\lambda ; t+t^{\prime}, t^{\prime}\right) H\left(\lambda ; t^{\prime}, s\right) \xi\right\|_{L^{2}(E)} \\
\leqq C_{0}\left[\left(t+t^{\prime}-s\right)^{3 / 2}-t^{3 / 2}+\left(t^{\prime}-s\right)^{3 / 2}\right]\|\xi\|_{L^{2}(E)},
\end{gathered}
$$

with some constant $C_{0}=C_{0}(\lambda ; T)$.

We devide a closed interval $[0, t], 0<t \leqq T$, into subintervals, i.e.

$$
\sigma_{N}: 0=t_{0}<t_{1}<\cdots<t_{N-1}<t_{N}=t, \quad t_{j}=(j / N) t, \quad j=0,1, \cdots, N .
$$

And we define the operator

$$
H\left(\lambda ; \sigma_{N} \mid t\right)=H\left(\lambda ; t, t_{N-1}\right) \cdots H\left(\lambda ; t_{1}, 0\right) .
$$

Combining with Proposition 4.1 and the similar argument as in Lemma 4.6, [13], we have the following, which is the part (b) in Theorem 1.2 (Cf. [6]).

Proposition 4.2. Assume that $(M, g)$ satisfies (A.0)-(A.2). Then, $\left\{H\left(\lambda ; \sigma_{N} \mid t\right)\right\}$ forms a Cauchy sequence in $\mathscr{B}\left(L^{2}(E)\right)$. Therefore, there exists a $C^{0}$ semi-group $\boldsymbol{H}(\lambda ; t), t>0$, in $L^{2}(E)$ such that for any $t>0$,

$$
\lim _{N \rightarrow \infty}\left\|\boldsymbol{H}(\lambda ; t)-H\left(\lambda ; \sigma_{N} \mid t\right)\right\|_{\mathscr{B}\left(L^{2}(E)\right)}=0,
$$

Moreover, there exists a positive constant $C_{0}^{\prime}=C_{0}^{\prime}(; T)$ such that

$$
\left\|\boldsymbol{H}(\lambda ; t)-H\left(\lambda ; \sigma_{N} \mid t\right)\right\|_{\mathscr{B}\left(L^{2}(E)\right)} \leqq C_{0}^{\prime} t N^{-1 / 2}\left(\exp \left(C_{3} t^{1 / 2}\right)\right) .
$$

Remark. By a slight modification of the above, we can generalize Proposition 4.2 for arbitrary subdivision of $[0, t]$ (Cf. [13] and [10]).

\section{$\S 5$. Computation of the infinitesimal generator.}

To finish the proof of Theorem 1.2, we only compute the infinitesimal generator of $\boldsymbol{H}(\lambda ; t)$. Namely, we get 
Proposition 5.1. Assume that $(M, g)$ satısfies (A.0)-(A.2). Then,

$$
(\partial / \partial t) \boldsymbol{H}(\lambda ; t) \boldsymbol{\xi}(x)=\lambda^{-1}\left[(1 / 2) \Delta_{x}^{D}-(1 / 12) \operatorname{Scal}_{g}(x)\right] \boldsymbol{H}(\lambda ; t) \xi(x)
$$

for $\xi \in C_{0}^{\infty}(E)$.

To prove the above proposition, we remark the following, which proved as same as in Lemma 4.2, [13],

LEMMA 5.2. Given $\xi \in C_{0}^{\infty}(E)$, we have

$$
(\partial / \partial t) \boldsymbol{H}(\lambda ; t) \xi(x)_{\mid t=0}=(\partial / \partial t) H(\lambda ; t, 0) \xi(x)_{1 t=0} .
$$

Since $\boldsymbol{H}(\lambda ; t)$ is a $C^{0}$ semi-group, it is sufficient for proving Proposition 5.1 to show the following:

Proposition 5.3. Under the same assumptions as in Proposition 5.1, we have for any $\xi \in C_{0}^{\infty}(E)$ and $x \in M$,

$$
H(\lambda ; t, 0) \xi(x)-\xi(x)=t \lambda^{-1} \mathcal{H}_{x} \xi(x)+t G(t ; \xi),
$$

where $\mathcal{H}_{x} \xi(x)=\left[(1 / 2) \Delta_{x}^{D}-(1 / 12) \operatorname{Scal}_{g}(x)\right] \xi(x)$ and $G(t ; \xi)$ satısfies

$$
\lim _{t \rightarrow 0}\|G(t ; \xi)\|_{L^{2}(E)}=0 \text {. }
$$

Proof. Recall (4.2). By using the integration by parts, we get

$$
\begin{aligned}
& (\partial / \partial t) H(\lambda ; t, 0) \xi(x)-\left(\lambda^{-1} / 2\right) H(\lambda ; t, 0) \Delta_{x}^{D} \xi(x) \\
& \quad=\lambda^{-1}\left(2 \pi \lambda^{-1} t\right)^{-m / 2} \int_{M}[\exp (-\lambda S(t, 0 ; x, y))] Q(x, y) \xi(y) d \mu_{g}(y),
\end{aligned}
$$

where

$$
\begin{aligned}
Q(x, y)= & -(1 / 2) \Delta_{y} \rho(x, y) P(x, y)+\left\langle\nabla_{y} \rho(x, y), D_{y} P(x, y)\right\rangle_{y} \\
& +(1 / 2) \rho(x, y) \Delta_{y}^{D} P(x, y) .
\end{aligned}
$$

Noticing that $\Delta_{y} \rho(x, y)_{1 y=x}=(1 / 6) \operatorname{Scal}_{g}(x)$ (Cf. [3], [13]), we have

where

$$
\begin{aligned}
\lambda \tilde{G}(t ; \xi)= & (\partial / \partial t) H(\lambda ; t, 0) \xi(x)-(1 / 2) H(\lambda ; t, 0) \Delta^{D} \xi(x) \\
& +(1 / 12) \operatorname{Scal}_{g}(x) \xi(x),
\end{aligned}
$$

$$
\begin{aligned}
\tilde{G}(t ; \xi)=(1 / 2)[H(\lambda ; t, 0)-I] \xi(x) \\
-(1 / 2)\left(2 \pi \lambda^{-1} t\right)^{-m / 2} \int_{M}[\exp (-\lambda S(t, 0 ; x, y))] \\
\times\left[q_{1}(x, y)+q_{2}(x, y)\right] \xi(y) d \mu_{g}(y),
\end{aligned}
$$




$$
\left\{\begin{array}{l}
q_{1}(x, y)=\left[\Delta_{y} \rho(x, y)-\Delta_{y} \rho(x, y)_{\mid x=y}\right] P(x, y), \\
q_{2}(x, y)=2\left\langle\nabla_{y} \rho(x, y), D_{x} P(x, y)\right\rangle+\rho(x, y) \Delta_{y} P(x, y)
\end{array}\right.
$$

Using Proposition 3.2, we have, for some constant $C_{4}=C_{4}(\lambda ; T)>0$,

$$
\begin{aligned}
& \left\|\boldsymbol{H}(\lambda ; t, 0) \Delta^{D} \xi(x)-\Delta^{D} \xi(x)\right\|_{L^{2}(E)} \\
& \quad \leqq C_{4} t^{1 / 2} \sup _{x \in M}\left[|\nabla \xi|_{x}+|\xi|_{x}\right]+O(t ; \xi),
\end{aligned}
$$

and

$$
\lim _{t \rightarrow 0+} O(t ; \xi)=0 .
$$

Also, by Proposition 2.2-2.6, we have

$$
\left|q_{i}(x, y)\right|_{(x, y) \leqq} k_{9} \exp \left(k_{9} d(x, y)\right), \quad i=1,2 .
$$

for any $x, y \in M$ with some constant $k_{9}>0$. Then, we get for some constant $C_{5}=C_{5}(\lambda ; T)$,

$$
\|G(t ; \xi)\|_{L^{2}(E)} \leqq\left[C_{5} t^{1 / 2} \exp \left(k_{9} t^{1 / 2}\right)\right]\|\xi\|_{L^{2}(E)} .
$$

Remarking $H(\lambda ; t, 0) \xi(x)-\xi(x)=\int_{0}^{t}(d / d \sigma) H(\lambda ; \sigma, 0) \xi(x) d \sigma$, we have the desired results.

Proposition 5.3 gives the part (c) in Theorem 1.2. By $\S \S 4-5$, we finish to prove Theorem 1.2 completely.

Now, for a later use in $\S 6$, we prepare the following properties : Let $\xi(\tau, y)$ be a mapping from $[s, t] \times M$ into $E$ which satisfies

(i) $\xi(\tau, \cdot) \in C(E)$ for each fixed $\tau \in[s, t]$.

(ii) $\xi(\tau, y)$ is Hölder continuous in $[s, T) \times M$.

(iii) Given any closed interval $\left[s_{1}, t_{1}\right] \subset[s, t], \xi(\tau, y)$ is bounded on $\left[s_{1}, t_{1}\right]$ $\times M$.

(iv) For any $t \in[s, T), \int_{s}^{t} d \tau \int_{M}|\xi(\tau, y)|_{y} d \mu_{g}(y)<+\infty$.

Proposition 5.4. Assume that $(M, g)$ satisfies (A.0)-(A.2). Let $\xi(\tau, y)$ be as above. Put $\xi(t, \tau ; x)$ and $\boldsymbol{\Xi}(t, x)$ by

$$
\begin{aligned}
& \xi(t, \tau ; x)=\int_{M} H(\lambda ; t, \tau ; x, y) \xi(\tau ; y) d \mu_{g}(y), \\
& \boldsymbol{E}(t, x)=\int_{s}^{t} \xi(t, \tau ; x) d \tau,
\end{aligned}
$$

Then, there exists a positive constant $C_{6}$ depending only the closed interval $\left[s_{1}, t_{1}\right]$ such that

$$
|(\partial / \partial t) \xi(t, \tau ; x)|_{x} \leqq C_{6}(t-\tau)^{-(1-r / 2)}, \quad s_{1} \leqq \tau<t<t_{1},
$$


where $\gamma$ is the Hölder exponent of $\xi$ at $(t, x)$. Also, in (5.14) the same inequalities replacing $\partial / \partial t$ by $D_{x}$ and $\Delta_{x}^{D}$ hold. Moreover, we have

$$
\mathscr{A}_{x} \boldsymbol{\Xi}(t, x)=\int_{s}^{t} \mathscr{H}_{x} \xi(t, \tau ; x) d \tau
$$

and

$$
(\partial / \partial t) \xi(t, x)=\xi(t, s)+\int_{s}^{t}(\partial / \partial t) \xi(t, \tau ; x) d \tau
$$

Proof. Given any $(t, x) \in[s, T) \times M$, let $\gamma$ be the Hölder exponent of $\xi$ at this point. Take a closed interval $\left[s_{1}, t_{1}\right]$ such that $s<s_{1}<t_{1}<T$. Then, there exists a positive constant $C_{6}^{\prime}$ and $\delta, 0<\delta<1$, such that $s_{1}<t-\delta$ and if $|t-\tau|<\delta$ and $d(x, y)<\delta$, then

$$
|P(x, y) \xi(\tau, y)-\xi(t, x)|_{x} \leqq C_{6}^{\prime}\left(|t-\tau|^{r}+(d(x, y))^{r}\right)
$$

and if $t-\delta<\tau<t<t^{\prime}$, then

$$
\int_{M}\left(\partial / \partial t^{\prime}\right) H\left(\lambda ; t^{\prime}, \tau ; x, y\right) \xi(\tau, y) d \mu_{g}(y)=-I_{1}-I_{2}+I_{3},
$$

where

$$
\begin{aligned}
& I_{1}=\int_{d(x, y) \leq \delta}\left(\partial / \partial t^{\prime}\right) H\left(\lambda ; t^{\prime}, \tau ; x, y\right)[P(y, x) \xi(t, x)-\xi(\tau, y)] d \mu_{g}(y), \\
& I_{2}=\int_{d(x, y) \geqq \delta}\left(\partial / \partial t^{\prime}\right) H\left(\lambda ; t^{\prime}, \tau ; x, y\right)[P(y, x) \xi(t, x)-\xi(\tau, y)] d \mu_{g}(y), \\
& I_{3}=\int_{M} H\left(\lambda ; t^{\prime}, \tau ; x, y\right)_{y}^{\#} P(y, x) \xi(t, x) d \mu_{g}(y) .
\end{aligned}
$$

So, there exists a constant $C_{6}^{\prime \prime}>0$ such that

$$
\left|I_{1}\right|_{x} \leqq C_{6}^{\prime \prime}(t-\tau)^{-(1-\gamma / 2)}, \quad\left|I_{2}\right|_{x} \leqq C_{6}^{\prime \prime}, \quad\left|I_{3}\right|_{x} \leqq C_{6}^{\prime \prime}(t-\tau)^{-1 / 2}
$$

which implies if $t-\delta \leqq t^{\prime}$, then

$$
|(\partial / \partial t) \xi(\lambda ; t, \tau ; x)|_{x} \leqq C_{6}^{\prime \prime \prime}\left(t^{\prime}-\tau\right)^{-(1-\gamma / 2)}
$$

with some constant $C_{6}^{\prime \prime \prime}>0$. On the other hand, if $s_{1} \leqq \tau \leqq t-s$, then $t^{\prime}-\tau \geqq \dot{\delta}>0$. Therefore, we see that $\left(\partial / \partial t^{\prime}\right) \xi\left(\lambda ; t^{\prime}, \tau ; x\right)$ is uniformly bounded in $\left(t^{\prime}, \tau, x\right)$, because of the form $(\partial / \partial t) H\left(\lambda ; t^{\prime}, \tau ; x, y\right)$ and (iii) of the properties of $\xi$. So, we have the estimate (5.16). Other estimates are obviously obtained. Now, by properties (iii) and (iv) of $\xi(\tau, x)$, we have for some constant $C_{7}$,

$$
\begin{aligned}
& \left|D_{x} \xi(\lambda ; t, \tau ; x)\right|_{x} \leqq C_{7}(t-\tau)^{-(1-\gamma / 2)} \\
& \left|\Delta_{x}^{D} \xi(\lambda ; t, \tau ; x)\right|_{x} \leqq C_{7}(t-\tau)^{-(1-\gamma / 2)}, \quad s_{1} \leqq \tau \leqq t<t_{1} .
\end{aligned}
$$


Remarking $\int_{s}^{t}(t-\tau)^{-(1-r / 2)} d \tau<+\infty$, we can interchanging the operator $\mathscr{H}_{x}$ and the integral. So, we have (5.15). Similarly, we get (5.16).

As a direct consequence of Proposition 5.4, and Lemma 3.3, we have

COROLLARY 5.5. Under the same notations and assumptions as in Proposition 5.4, we have

$$
\begin{gathered}
\mathscr{H}_{x} \boldsymbol{\Xi}(t, x)=\int_{s}^{t} d \tau \int_{M} \mathscr{H}_{x} H(\lambda ; t, \tau ; x, y) \xi(\tau, y) d \mu_{g}(y), \\
(\partial / \partial t) \boldsymbol{\Xi}(t, x)=\xi(t, x)+\int_{s}^{t} d \tau \int_{M}(\partial / \partial t) H(\lambda ; \tau, s ; x, y) \xi(\tau, y) d \mu_{g}(y) .
\end{gathered}
$$

\section{$\S 6$. Construction of the fundamental solution.}

To prove the main theorem stated in the introduction, we shall construct the fundamental solution for the following system of parabolic equations :

$$
(\partial / \partial t) \xi(t, x)=\lambda^{-1} \mathscr{H}_{x} \xi(t, x), \quad \xi(0, x)=\xi_{0}(x) \in C_{0}(E),
$$

where $\mathscr{H}_{x}=(1 / 2) \Delta_{x}^{D}-(1 / 12) \operatorname{Scal}_{g}(x)$.

Throughout this section, we assume the assumptions (A.0)-(A.2). We denote by $L$ the differential operator $-(\partial / \partial t)+\lambda^{-1} \mathscr{H}_{x}$. Recall the kernel function $H(\lambda ; t, s ; x, y)$ of $H(\lambda ; t, s), 0 \leqq s<t<T$ in (4.1). Set

$$
\begin{aligned}
J_{0}(\lambda ; t, s ; x, y) & =L H(\lambda ; t, s ; x, y) \\
& =-\left[(\partial / \partial t)-\lambda^{-1} \mathscr{H}_{x}\right] H(\lambda ; t, s ; x, y) .
\end{aligned}
$$

Lemma 6.1. For any $0 \leqq s<t<T$ and $x, y \in M$, there exists positive constants $M_{0}, k_{6}$ and $M_{1}=M_{1}(\lambda ; T)$ such that

and

$$
\begin{aligned}
& |H(\lambda ; t, s ; x, y)|_{(x, y)} \\
& \quad \leqq M_{0} \lambda^{m / 2}(t-s)^{-m / 2} \exp \left[-\lambda\left(d^{2}(x, y) /(2(t-s))\right]\right.
\end{aligned}
$$

where $\varepsilon^{*}=1-\varepsilon$.

$$
\begin{aligned}
&\left|J_{0}(\lambda ; t, s ; x, y)\right|_{(x, y)} \\
& \leqq M_{0}\left(M_{1} / \varepsilon\right)\left(\exp \left(k_{6} T / \varepsilon \lambda\right)\right) \lambda^{m / 2-2}(t-s)^{-(m-1) / 2} \\
& \times \exp \left[-\lambda\left(\varepsilon^{*} d^{2}(x, y) /(2(t-s))\right],\right.
\end{aligned}
$$

Proof. (6.3) is easily obtained by the form (3.1) and Lemma 2.4. For (6.4), we have by (4.1) 


$$
\begin{aligned}
& \left|J_{0}(\lambda ; t, s ; x, y)\right|(x, y) \\
& \leqq M_{0} \lambda^{m / 2-1} k_{5}(t-s)^{-m / 2} d(x, y) \\
& \quad \times \exp \left[-\lambda\left(d^{2}(x, y) /(2(t-s))-k_{5} d(x, y)\right)\right]
\end{aligned}
$$

where $k_{5}$ is a positive constant. Putting the function $F(r), r \geqq 0$, by

$$
F(r)=r\left[\exp \left(k_{5} r-\lambda \varepsilon r^{2} /(2(t-s))\right],\right.
$$

we have

$$
\begin{aligned}
F(r) \leqq & (2 \lambda \varepsilon)^{-1}(t-s)^{1 / 2}\left[k_{5}(t-s)^{1 / 2}+\left(\left(k_{5}\right)^{2}(t-s)+4 \lambda \varepsilon\right)^{1 / 2}\right] \\
& \left.\times\left[\exp \left((4 \lambda \varepsilon)^{-1}\left(k_{5}(t-s)+\left(\left(k_{5}\right)^{2}(t-s)+4 \lambda \varepsilon\right)\right)^{1 / 2}(t-s)^{1 / 2}\right)\right)\right] \\
\leqq & C_{8}^{\prime} \lambda^{-1} \varepsilon^{-1}\left[\exp \left(k_{6} T / \varepsilon \lambda\right)\right](t-s)^{1 / 2}
\end{aligned}
$$

with some constants $C_{8}^{\prime}=C_{8}^{\prime}(\lambda ; T)$ and $k_{6}$. Substituting (6.6) into (6.5), we get, for any $x, y \in M$,

$$
\begin{aligned}
& \left|J_{0}(\lambda ; t, s ; x, y)\right|_{(x, y)} \\
& \leqq M_{0} C_{8}^{\prime} \lambda^{m / 2-2} \varepsilon^{-1}\left[\exp \left(k_{6} T / \varepsilon \lambda\right)\right](t-s)^{-(m-1) / 2} \\
& \quad \times \exp \left[-\lambda\left(\varepsilon^{*} d^{2}(x, y) /(2(t-s))\right]\right.
\end{aligned}
$$

So, we get Lemma 6.1 .

Now, we put

$$
J_{1}(\lambda ; t, s ; x, y)=\int_{s}^{t} d \sigma \int_{M} J_{0}(\lambda ; t, \sigma ; x, z)_{z}^{\#} J_{0}(\lambda ; \sigma, s ; z, y) d \mu_{g}(z) .
$$

LEMMA 6.2. For any $0 \leqq s<t<T$, and $x, y \in M$, there exists positive constants $M_{2}=M_{2}(\lambda ; T)$ such that for any $0<\varepsilon<1 / 2$,

$$
\begin{aligned}
& \left|J_{1}(\lambda ; t, s ; x, y)\right|_{(x, y)} \\
& \leqq M_{0}\left(M_{2} / \varepsilon\right)^{2}\left(\varepsilon^{*}\right)^{-m / 2}\left[\exp \left(k_{6} T / \varepsilon \lambda\right)\right] \lambda^{-m / 2-2}(t-s)^{-(m / 2)+2} \\
& \quad \times B(3 / 2: 3 / 2)\left[\exp \left(-\lambda\left(\varepsilon^{* *} d^{2}(x, y) /(2(t-s))\right)\right],\right.
\end{aligned}
$$

where $\varepsilon^{* *}=1-2 \varepsilon$, and $B(:)$ is the Beta function.

Proof. First, we put

$$
J_{1}(\lambda ; t, s, \sigma ; x, y)=\int_{M} J_{0}(\lambda ; t, \sigma ; x, z)_{2}^{\#} J_{0}(\lambda ; \sigma, s ; z, y) d \mu_{g}(z) .
$$

By the comparison theorem, we have $d^{2}(z, y) \geqq|Z-Y|^{2}$, if we can write $y=$ $\operatorname{Exp}_{x} Y$ and $z=\operatorname{Exp}_{x} Z$. Thus, we get 


$$
\begin{aligned}
& \left|J_{1}(\lambda ; t, s, \sigma ; x, y)\right|_{(x, y)} \\
& \leqq M_{0}^{2} M_{1}^{2}\left[\exp \left(k_{6} T / \varepsilon \lambda\right)\right]^{2} \lambda^{m-4} \varepsilon^{-2}(t-\sigma)^{-(m-1) / 2}(\sigma-s)^{-(m-1) / 2} \\
& \quad \times \int_{T_{x} M} \exp -\left(\lambda \left(\varepsilon^{*}|Z|^{2} /(2(t-\sigma))\right.\right.
\end{aligned}
$$

with some constant $k_{5}>0$. Since

$$
\left.\left.+\varepsilon^{*}|Z-Y|^{2} /(2(\sigma-s))\right)-k|Z|\right) d Z \text {, }
$$

$$
\begin{aligned}
& |Z|^{2} /(2(t-\sigma))+|Z-Y|^{2} /(2(\sigma-s)) \\
& =(t-s) /(2(t-\sigma)(\sigma-s))|Z-((t-\sigma) /(t-s)) Y|^{2} \\
& \quad+(1 /(2(t-s)))|Y|^{2},
\end{aligned}
$$

we have

$$
\begin{aligned}
& \left|J_{1}(\lambda ; t, s, \sigma ; x, y)\right|_{(x, y)} \\
& \leqq \\
& \quad M_{0}^{2}\left(M_{1} / \varepsilon\right)^{2}\left[\exp \left(2 k_{6} T / \varepsilon \lambda\right)\right] \lambda^{m-4}\left(2 / \lambda \varepsilon^{*}\right)^{m / 2}(t-s)^{-m / 2} \\
& \quad \times(t-\sigma)^{1 / 2}(\sigma-s)^{1 / 2}\left[\exp -\left(\left(\lambda \varepsilon^{*} d^{2}(x, y) /(2(t-s))-k_{5} d(x, y)\right)\right]\right. \\
& \quad \times \int_{T_{x} M} \exp \left(-\left(\left|Z^{\prime}\right|^{2}-k_{5}\left(2(t-\sigma)(\sigma-s) /\left(\lambda \varepsilon^{*}(t-s)\right)\right)^{1 / 2}\left|Z^{\prime}\right|\right) d Z^{\prime}\right.
\end{aligned}
$$

because of $0<(t-\sigma) /(t-s)<1$. By an easy computation, we get

$$
\begin{aligned}
& \left|J_{1}(\lambda ; t, s, \sigma ; x, y)\right|_{(x, y)} \\
& \leqq \\
& \quad(\pi / 2)^{1 / 2}\left(2^{m / 2}\right) M_{0}^{2}\left(M_{1} / \varepsilon\right)^{2}\left(\varepsilon^{*}\right)^{-m / 2} \lambda^{(m / 2)-4}\left[\exp \left(2 k_{6} T / \varepsilon \lambda\right)\right] \\
& \quad \times(t-\sigma)^{1 / 2}(\sigma-s)^{1 / 2}(t-s)^{-m / 2} \operatorname{vol}\left(S^{m-1}\right)\left[\exp (t-s) k_{5}^{2} / 8 \lambda \varepsilon^{*}\right] \\
& \quad \times\left[\exp \left(-\lambda\left(\varepsilon^{*} d^{2}(x, y) /(2(t-s))-k_{5} d(x, y)\right)\right)\right] .
\end{aligned}
$$

Therefore, there exists positive constants $M_{2}$ and $k_{7}$ such that we

$$
\begin{aligned}
&\left|J_{1}(\lambda ; t, s ; x, y)\right|_{(x, y)} \\
& \leqq M_{0}^{2}\left(M_{1} / \varepsilon\right)^{2}\left(\varepsilon^{*}\right)^{-m / 2} \lambda^{(m / 2)-4}[\exp (k T / \varepsilon \lambda)]^{2}(t-s)^{-m / 2} \\
& \quad \times\left[\exp \left(-\lambda\left(\varepsilon^{* *} d^{2}(x, y) /(2(t-s))\right)\right] \int_{s}^{t}(t-\sigma)^{1 / 2}(\sigma-s)^{1 / 2} d \sigma\right. \\
& \leqq M_{0}^{2}\left(M_{2} / \varepsilon\right)^{2}\left(\varepsilon^{*}\right)^{-m / 2} \lambda^{(m / 2)-4}\left[\exp \left(k_{7} T / \varepsilon \lambda\right)\right]^{2}(t-s)^{-(m-4) / 2} \\
& \times B(3 / 2 ; 3 / 2)\left[\exp \left(-\lambda\left(\varepsilon^{* *} d^{2}(x, y) /(2(t-s))\right)\right],\right.
\end{aligned}
$$

where $\varepsilon^{* *}=1-2 \varepsilon$, which gives Lemma 6.2.

Successively, we define, for $n \geqq 1$,

$$
J_{n}(\lambda ; t, s ; x, y)=\int_{s}^{t} d \sigma \int_{M} J_{0}(\lambda ; t, \sigma ; x, z)_{z}^{\#} J_{n-1}(\lambda ; \sigma, s ; z, y) d \mu_{g}(z) .
$$

By a similar computation as above, we get 
LEMMA 6.3. For any $0 \leqq s<t<T$ and $x, y \in M$, the following estimate holds: Given any $0<\varepsilon<1 / 2$,

$$
\begin{aligned}
& \left|J_{n}(\lambda ; t, s ; x, y)\right|_{(x, y)} \\
& \leqq \\
& \quad M_{0}^{n+1}\left(M_{2} / \varepsilon\right)^{n+1}\left[\exp \left(k_{7} T / \varepsilon \lambda\right)\right]^{n+1}\left(\varepsilon^{*}\right)^{-m+n / 2}(t-s)^{-(m-1-3 n) / 2} \\
& \quad \times \lambda^{m / 2-2(n+1)} \prod_{a=1}^{n} B(3 / 2: 3(a+1) / 2)\left[\exp \left(-\lambda\left(\varepsilon^{* *} d^{2}(x, y) /(2(t-s))\right)\right]\right.
\end{aligned}
$$

where $\varepsilon^{* *}=1-2 \varepsilon$.

$$
(n \geqq 1) \text {, }
$$

Remark that

$$
\prod_{a=1}^{n} B(3 / 2: 3(a+1) / 2)=\Gamma(3 / 2)^{n} / \Gamma(3(n+1) / 2) \leqq k_{8}^{n} / n ! .
$$

for some positive constant $k_{8}$. Then, there exists positive constants $M_{3}=M_{3}(\lambda ; T)$ and $k_{9}$ such that

$$
\begin{aligned}
& \sum_{n=0}^{\infty}\left|J_{n}(\lambda ; t, s ; x, y)\right|_{(x, y)} \\
& \leqq M_{0} M_{3} \lambda^{m / 2-2}\left[\exp \left(\left(M_{3} \varepsilon^{-1} \lambda^{-2}\left(\varepsilon^{*}\right)^{-m / 2}\right) \exp \left(k_{9} T / \lambda \varepsilon\right)\right)\right](t-s)^{-(m-1) / 2} \\
& \times\left[\exp M_{3}(t-s)^{3 / 2}\right]\left[\exp -\lambda\left(\varepsilon^{* *} d^{2}(x, y) /(2(t-s))\right)\right]
\end{aligned}
$$

Thus, on $\{(t-s) \mid 0 \leqq s<t<T\} \times M \times M$, we can define a function

$$
K(\lambda ; t, s ; x, y)=\sum_{n=0}^{\infty} J_{n}(\lambda ; t, s ; x, y)
$$

and for any $C>1$, on $\left\{(t, s) \mid 0 \leqq s<t<T, C^{-1}<t-s<C\right\} \times M \times M$, the infinite sum of (6.17) converges uniformly on each compact set, and we have

$$
\begin{aligned}
&|K(\lambda ; t, s ; x, y)|_{(x, y)} \\
& \leqq M_{0} M_{3} \lambda^{m / 2-2}\left[\exp \left(M_{3} \varepsilon^{-1} \lambda^{-2}\left(\varepsilon^{*}\right)^{-m / 2} \exp \left(k_{9} T / \lambda \varepsilon\right)\right)\right]\left[\exp M_{3}(t-s)\right] \\
& \quad \times(t-s)^{-(m-1) / 2}\left[\exp -\lambda\left(\varepsilon^{* *} d^{2}(x, y) /(2(t-s))\right] .\right.
\end{aligned}
$$

Moreover, by a direct computation, we get

LEMMA 6.4. Let $J_{n}(\lambda ; t, s ; x, y)$ be the function defined by (6.14). For any $0 \leqq s<t<T$, there exist constants $M_{4}=M_{4}(\lambda ; T)>0$ and $k_{10}>0$ such that

$$
\begin{aligned}
& \sum_{n=0}^{\infty} \int_{M}\left|J_{n}(\lambda ; t, s ; x, y)\right|_{(x, y)} d \mu_{g}(x) \\
& \quad \leqq M_{0} M_{4} \lambda^{m / 2-2}\left[\exp \left(\left(M_{4} \varepsilon^{-1} \lambda^{-2}\left(\varepsilon^{*}\right)^{-m / 2} \exp \left(k_{10} T / \lambda \varepsilon\right)\right)\right]\left[\exp \left(M_{4}(t-s)\right)\right]\right. \\
& \sum_{n=0}^{\infty} \int_{M}\left|J_{n}(\lambda ; t, s ; x, y)\right|_{(x, y)} d \mu_{g}(y) \\
& \quad \leqq M_{0} M_{4} \lambda^{m / 2-2}\left[\exp \left(\left(M_{4} \varepsilon^{-1} \lambda^{-2}\left(\varepsilon^{*}\right)^{-m / 2}\right) \exp \left(k_{10} T / \lambda \varepsilon\right)\right)\right]\left[\exp \left(M_{4}(t-s)\right)\right]
\end{aligned}
$$


Therefore, we have

$$
\begin{aligned}
& \int_{M}|K(\lambda ; t, s ; x, y)|_{(x, y)} d \mu_{g}(y) \\
& \leqq M_{0} M_{4} \lambda^{m / 2-2}\left[\exp \left(\left(M_{4} \varepsilon^{-1} \lambda^{-2}\left(\varepsilon^{*}\right)^{-m / 2} \exp \left(k_{10} T / \lambda \varepsilon\right)\right)\right]\right. \\
& \times\left[\exp \left(M_{4}(t-s)\right)\right](t-s)^{1 / 2} \\
& \int_{M}|K(\lambda ; t, s ; x, y)|_{(x, y)} d \mu_{g}(x) \\
& \leqq M_{0} M_{4} \lambda^{m / 2-2}\left[\exp \left(\left(M_{4} \varepsilon^{-1} \lambda^{-2}\left(\varepsilon^{*}\right)^{-m / 2} \exp \left(k_{10} T / \lambda \varepsilon\right)\right)\right]\right. \\
& \times\left[\exp \left(M_{4}(t-s)\right)\right](t-s)^{1 / 2} .
\end{aligned}
$$

Now, fix $(s, y)$ and consider $\xi(t, z)=K(\lambda ; t, s ; x, z)$. Applying Corollary 5.5, we have

$$
\begin{aligned}
& \mathscr{H}_{x} \int_{s}^{t} d \sigma \int_{M} H(\lambda ; t, \sigma ; x, z)_{z}^{\#} K(\lambda ; \sigma, s ; z, y) d \mu_{g}(z) \\
& =\int_{s}^{t} d \sigma \int_{M} \mathcal{H}_{x} H(\lambda ; t, \sigma ; x, y)_{z}^{\#} K(\lambda ; \sigma, s ; z, y) d \mu_{g}(z),
\end{aligned}
$$

Thus, we get

$$
\begin{aligned}
& (\partial / \partial t) \int_{s}^{t} d \sigma \int_{M} H(\lambda ; t, \sigma ; x, z)_{z}^{\#} K(\lambda ; \sigma, s ; z, y) d \mu_{g}(z) \\
& =K(\lambda ; t, s ; x, y)+\int_{s}^{t} d \sigma \int_{M}(\partial / \partial t) H(\lambda ; t, \sigma ; x, z)_{z}^{\#} K(\lambda ; \sigma, s ; z, y) d \mu_{g}(z) .
\end{aligned}
$$

So, we have

$$
\begin{aligned}
& -\left[(\partial / \partial t)-\lambda^{-1} \mathcal{H}_{x}\right] \int_{s}^{t} d \sigma \int_{M} H(\lambda ; t, \sigma ; x, z)_{z}^{\#} K(\lambda ; \sigma, s ; z, y) d \mu_{g}(z) \\
& =K(\lambda ; t, s ; x, y)+\int_{s}^{t} d \sigma \int_{M} J_{0}(\lambda ; t, \sigma ; x, z)_{z}^{\#} K(\lambda ; \sigma, s ; z, y) d \mu_{g}(z) \\
& =-J_{0}(\lambda ; t, s ; x, y) .
\end{aligned}
$$

Therefore, we obtain the following:

PROPOSITION 6.5. Under the same assumptions and notations as above, put

$$
\begin{aligned}
& \boldsymbol{H}(\lambda ; t, s ; x, y) \\
& \quad=H(\lambda ; t, s ; x, y)+\int_{s}^{t} d \sigma \int_{M} H(\lambda ; t, \sigma ; x, z)_{z}^{\#} K(\lambda ; \sigma, s ; z, y) d \mu_{g}(z) .
\end{aligned}
$$

Then, the following properties hold:

(i) $\boldsymbol{H}(\lambda ; t, s ; x, y)$ is continuous in $\{(t, s) \mid 0 \leqq s<t<T\} \times M \times M$.

(ii) $\boldsymbol{H}(\lambda ; t, s ; x, y)$ satisfies 


$$
(\partial / \partial t) \boldsymbol{H}(\lambda ; t, s ; x, y)=\lambda^{-1} \mathscr{H}_{x} \boldsymbol{H}(\lambda ; t, s ; x, y)
$$

(iii) There exists a positive constant $M_{5}=M_{5}(\lambda ; T)$ such that

and

$$
\begin{aligned}
|\boldsymbol{H}(\lambda ; t, s ; x, y)|_{(x, y)} \\
\leqq M_{0} M_{5} \lambda^{m / 2}\left[\exp \left(\left(M_{5} \varepsilon^{-1} \lambda^{-2}\left(\varepsilon^{*}\right)^{-m / 2} \exp \left(k_{10} T / \lambda \varepsilon\right)\right)\right]\left[\exp \left(M_{5}(t-s)\right)\right]\right. \\
\times\left[\exp -\lambda\left(\varepsilon^{* *} d^{2}(x, y) /(2(t-s))\right]\right.
\end{aligned}
$$

$$
\begin{aligned}
& \int_{M}|\boldsymbol{H}(\lambda ; t, s ; x, y)|_{(x, y)} d \mu_{g}(x) \\
& \quad \leqq M_{0} M_{5} \lambda^{m / 2}\left[\exp \left(\left(M_{5} \varepsilon^{-1} \lambda^{-2}\left(\varepsilon^{*}\right)^{-m / 2} \exp \left(k_{10} T / \lambda \varepsilon\right)\right)\right]\left[\exp \left(M_{5}(t-s)\right)\right]\right. \\
& \int_{M}|\boldsymbol{H}(\lambda ; t, s ; x, y)|_{(x, y)} d \mu_{g}(y) \\
& \quad \leqq M_{0} M_{5} \lambda^{m / 2}\left[\exp \left(\left(M_{5} \varepsilon^{-1} \lambda^{-2}\left(\varepsilon^{*}\right)^{-m / 2} \exp \left(k_{10} T / \lambda \varepsilon\right)\right)\right]\left[\exp \left(M_{5}(t-s)\right)\right] .\right.
\end{aligned}
$$

(iv) Therefore, defining $\boldsymbol{H}(\lambda ; t, s) \xi(x)=\int_{M} \boldsymbol{H}(\lambda ; t, s ; x, y) \xi(y) d \mu_{g}(y)$, we have a bounded linear operator $\boldsymbol{H}(\lambda ; t, s)$ on $L^{2}(E)$ and is $C^{0}$ semi-group with infinitesimal generator $\lambda^{-1} \mathscr{H}$ in (6.26).

Proposition 6.5 shows that $\boldsymbol{H}(\lambda ; t, s ; x, y)$ defined by $(6.25)$ determines a fundamental solution for the parabolic equation (6.1).

Remark. Cheng et al. [5] gives the upper estimate for the heat kernel of Laplace-Beltrami operator acting on functions only by assuming the boundedness of the curvature tensor of $g$.

By a similar argument as in Lemma 4.4, we have

LEMMA 6.6. Let $\xi(\tau, z)$ be a bounded continuous mapping from $[s, t] \times M$ to $E$ such that for any fixed $\tau \in[s, t], \xi(\tau, \cdot) \in C(E)$. Then, the following equalities hold uniformly on any compact set on $M$ :

$$
\left\{\begin{array}{l}
\lim _{\tau \rightarrow s} \int_{M} \xi(\tau, z)_{z}^{\#} \boldsymbol{H}(\lambda ; \tau, s ; x, y) d \mu_{g}(y)=\xi(s, y), \\
\lim _{\tau \rightarrow t} \int_{M} \boldsymbol{H}(\lambda ; t, \tau ; z, y)_{z}^{\#} \xi(\tau, z) d \mu_{g}(z)=\xi(t, y) .
\end{array}\right.
$$

\section{$\S 7$. Convergence of the product integral as the kernel function.}

In this section, we shall prove Theorem 1.3, which gives the main theorem in the introduction, using the fundamental solution constructed in $\S 6$. Let $[0, t]$ be any closed interval such that $0<t<T$, and $T$ be any fixed positive number. Let $\sigma_{N}$ be a $N$-equal subdivision of $[0, t]$;

$$
\sigma_{N}: 0=t_{0}<t_{1}<\cdots<t_{N-1}<t_{N}=t, \quad t_{j}=(j / N) t .
$$


We define a operator $H\left(\lambda ; \sigma_{N} \mid t\right)$ associated with the subdivision $\sigma_{N}$ :

$$
H\left(\lambda ; \sigma_{N} \mid t\right)=H\left(\lambda ; t, t_{N-1}\right) H\left(\lambda ; t_{N-1}, t_{N-2}\right) \cdots H\left(\lambda ; t_{1}, 0\right),
$$

and we denote by $H\left(\lambda ; \sigma_{N} \mid t ; x, y\right)$ the kernel function of the operator (7.2), i.e.

$$
\begin{aligned}
H\left(\lambda ; \sigma_{N} \mid t ; x, y\right)= & \int_{M} \cdots \int_{M} H\left(\lambda ; t, t_{N-1} ; x, z_{N-1}\right) \frac{\#}{z_{N-1}} \cdots \\
& \ldots{\frac{z_{1}}{\#}}_{1} H\left(\lambda ; t_{1}, 0 ; z_{1}, y\right) d \mu_{g}\left(z_{N-1}\right) \cdots d \mu_{g}\left(z_{1}\right),
\end{aligned}
$$

where $H(\lambda ; t, s ; x, y)$ is defined in (4.1).

To prove Theorem 1.3, we need several steps as below. First, put

$$
R(\lambda ; t, s)=H(\lambda ; t, s)-\boldsymbol{H}(\lambda ; t, s)
$$

and denote by $R(\lambda ; t, s ; x, y)$ the kernel function of (7.4). Then, we get the following, which gives Corollary stated in $\S 0$ :

Proposition 7.1. For any $0<\varepsilon<1 / 2$, there exists a positive constant $\gamma_{1}=$ $\gamma_{1}(\lambda ; T, \varepsilon)$ such that

$$
\begin{aligned}
& |R(\lambda ; t, s ; x, y)|_{(x, y)} \\
& \quad \leqq M_{0} \gamma_{1}(t-s)^{-(m-3) / 2}\left[\exp -\lambda\left(\varepsilon^{* *} d^{2}(x, y) /(2(t-s))\right)\right]
\end{aligned}
$$

where $\varepsilon^{* *}=1-2 \varepsilon$.

Proof. Combining (6.25) with (7.4), we have

$$
\begin{aligned}
& |R(\lambda ; t, s ; x, y)|_{(x, y)} \\
& \leqq M_{0} \gamma_{1} \int_{s}^{t}(t-\sigma)^{-m / 2}(\sigma-s)^{-(m-1) / 2} \\
& \quad \times \int_{T_{x} M} \exp -\lambda\left(\varepsilon^{* *}\left(|Z|^{2} /(2(t-\sigma))-|Z-Y|^{2} /(2(\sigma-s))\right) d Z d \sigma\right. \\
& \leqq M_{0} \gamma_{1}(t-s)^{-(m-3) / 2}\left[\exp -\lambda\left(\varepsilon^{* *} d^{2}(x, y) /(2(t-s))\right],\right.
\end{aligned}
$$

by a similar computations as in $\S 6$. Thus, we get (7.5).

Now, we obtain

$$
\begin{aligned}
H\left(\lambda ; \sigma_{N} \mid t\right)-\boldsymbol{H}(\lambda ; t) \\
=H\left(\lambda ; t, t_{N-1}\right) \cdots H\left(\lambda ; t_{1}, 0\right)-\boldsymbol{H}(\lambda ; t) \\
=\left[\boldsymbol{H}\left(\lambda ; t, t_{N-1}\right)+R\left(\lambda ; t, t_{N-1}\right)\right] \cdots \\
\quad \quad \cdots\left[\boldsymbol{H}\left(\lambda ; t_{1}, 0\right)+R\left(\lambda ; t_{1}, 0\right)\right]-\boldsymbol{H}(\lambda ; t, 0) .
\end{aligned}
$$

Using the evolutional property of $\boldsymbol{H}(\lambda ; t, s)$, we shall write down the right hand side of (7.7). Let 
(7.8)

$$
\mathscr{P}=\left\{\begin{array}{c}
\left(\alpha_{1}, \cdots, \alpha_{k} ; \beta_{1}, \cdots, \beta_{k+1}\right) ; k=1, \cdots, N, \alpha_{\imath}>0, \beta_{i} \geqq 0, \\
\sum_{i=1}^{k}\left[\alpha_{i}+\beta_{\imath}\right]+\beta_{k+1}=N
\end{array}\right\} .
$$

Also, we denote by

$$
\begin{array}{r}
A_{j}=\alpha_{1}+\cdots+\alpha_{\jmath}, \quad B_{j}=\beta_{1}+\cdots+\beta_{\jmath} \\
j \geqq 1, \quad A_{0}=0, \quad B_{0}=0 .
\end{array}
$$

Thus, $A_{k}+B_{k}=N$. The right hand side of (7.7) is written by

where

$$
\begin{aligned}
& H\left(\lambda ; \sigma_{N} \mid t\right)-\boldsymbol{H}(\lambda ; t) \\
& \quad=\sum_{\left(\alpha_{1}, \cdots, \alpha_{k} ; \beta_{1}, \cdots, \beta_{k+1}\right) \in \mathscr{P}} I\left(\alpha_{1}, \cdots, \alpha_{k} ; \beta_{1}, \cdots, \beta_{k+1}\right)
\end{aligned}
$$

$$
\begin{aligned}
& \mathscr{I}\left(\alpha_{1}, \cdots, \alpha_{k} ; \beta_{1}, \cdots, \beta_{k+1}\right) \\
& =\boldsymbol{H}\left(\lambda ; t,\left(A_{k}+B_{k}\right) t / N\right) \\
& \quad \times R\left(\lambda ;\left(A_{k}+B_{k}\right) t / N,\left(A_{k}-1+B_{k}\right) t / N\right) \cdots \\
& \quad \cdots R\left(\lambda ;\left(A_{k-1}+1+B_{k}\right) t / N,\left(A_{k-1}+B_{k}\right) t / N\right) \\
& \quad \times \boldsymbol{H}\left(\lambda ;\left(A_{k-1}+B_{k}\right) t / N,\left(A_{k-1}+B_{k-1}\right) t / N\right) \\
& \quad \times R\left(\lambda ;\left(A_{1}+B_{1}\right) t / N,\left(A_{1}-1+B_{1}\right) t / N\right) \cdots \\
& \quad \cdots R\left(\lambda ;\left(1+B_{1}\right) t / N, B_{1} t / N\right) \\
& \quad \times \boldsymbol{H}\left(\lambda ; B_{1} t / N, 0\right) .
\end{aligned}
$$

Now, we put

$$
\begin{aligned}
R^{(j)}\left(\lambda ; A_{\jmath}, B_{j} \mid t\right)= & R\left(\lambda ;\left(A_{\jmath}+B_{j}\right) t / N,\left(A_{j}-1+B_{j}\right) t / n\right) \cdots \\
& \cdots R\left(\lambda ;\left(A_{\jmath-1}+1+B_{j}\right) t / N,\left(A_{\jmath-1}+B_{j}\right) t / N\right)
\end{aligned}
$$

and denote by $R^{(j)}\left(\lambda ; A_{\jmath}, B_{j} \mid t ; x, y\right)$ the kernel function of (7.12).

LEMMA 7.2. Given any $0<\varepsilon<1 / 3$, there exists a positive constant $\gamma_{2}=\gamma_{2}(\lambda ; T, \varepsilon)$ and $\gamma_{3}=\gamma_{3}(\lambda ; T, \varepsilon)$ such that

$$
\begin{aligned}
& \left|R^{(j)}\left(\lambda ; A_{\jmath}, B_{\jmath}, t ; x, y\right)\right|_{(x, y)} \\
& \leqq M_{0}^{\alpha} \gamma_{2}^{\alpha}{ }_{2}\left[\exp \left(\gamma_{3}\left(\alpha_{j} / N\right) t\right](t / N)^{3 \alpha_{j} / 2}\left(\alpha_{j} t / N\right)^{-m / 2}\right. \\
& \times\left[\exp -\lambda\left(\varepsilon^{* * *} d^{2}(x, y) /\left(2 t_{j} / N\right)\right)\right]
\end{aligned}
$$

where $\varepsilon^{* * *}=1-3 \varepsilon$.

Proof. Generally, take $t_{1}, \cdots, t_{a} \in[0, t), o \leqq t_{1}<\cdots<t_{a}<t$, and put

$$
R\left(\lambda ; t_{a}, \cdots, t_{1}\right)=R\left(\lambda ; t_{a}, t_{a-1}\right) \cdots R\left(\lambda ; t_{2}, t_{1}\right) .
$$


We denote by $R\left(\lambda ; t_{a}, \cdots, t_{1} ; x, y\right)$ the kernel function of (7.14). To prove (7.13), it is sufficient to get the following estimate for (7.14):

$$
\begin{aligned}
& \left|R\left(\lambda ; t_{a}, \cdots, t_{1}\right)\right|_{(x, y)} \\
& \leqq M_{0}^{a-1} \gamma_{2}^{a-1}\left[\exp \left(\gamma_{3}\left(t_{a}-t_{1}\right)\right)\right] \prod_{k=1}^{a-1}\left(t_{k+1}-t_{k}\right)^{3 / 2}\left(t_{a}-t_{1}\right)^{-m / 2} \\
& \quad \times\left[\exp -\lambda\left(\varepsilon^{* * *} d^{2}(x, y) /\left(2\left(t_{a}-t_{1}\right)\right)\right)\right] .
\end{aligned}
$$

We shall show (7.15) by induction. Remark that (7.15) holds for $a=1$ easily by Lemma 7.1. Assume that (7.15) holds for $a-1 \geqq 1$. Then, by a similar computation as in Lemma 7.1, we have

$$
\begin{aligned}
& \left|R\left(\lambda ; t_{a}, \cdots, t_{1}\right)\right|_{(x, y)} \\
& \qquad M_{0}^{a-1} \gamma_{2}^{a-2}\left[\exp \left(\gamma_{3}\left(t_{a-1}-t_{1}\right)\right) \prod_{k=1}^{a-2}\left(t_{k+1}-t_{k}\right)^{3 / 2}\left(t_{a}-t_{a-1}\right)^{-(m-3) / 2}\right. \\
& \quad \times\left(t_{a}-t_{1}\right)^{-m / 2} \int_{T_{x} M} \exp -\left(\lambda \varepsilon ^ { * * * } \left(|Z|^{2} /\left(2\left(t_{a}-t_{a-1}\right)\right.\right.\right. \\
& \left.\quad-|Z-Y|^{2} /\left(2\left(t_{a}-t_{1}\right)\right)\right) d Z
\end{aligned}
$$

because of $\exp -\left(\lambda \varepsilon|Z|^{2} /\left(2\left(t_{a}-t_{a-1}\right)\right)-k|Z|\right) \leqq \exp \left(\left(t_{a}-t_{a-1}\right) k^{2} / 2 \lambda \varepsilon\right.$. By (7.16), we get

$$
\begin{aligned}
& \left|R\left(\lambda ; t_{a}, \cdots, t_{1}\right)\right|_{(x, y)} \\
& \leqq \\
& \left.\quad M_{1}^{a} \gamma_{2}^{a-2} \gamma_{1}\left[\exp \gamma_{3}\left(t_{a}-t_{1}\right)\right)\right] \prod_{k=1}^{a-1}\left(t_{k+1}-t_{k}\right)^{3 / 2}\left(t_{a}-t_{1}\right)^{-m / 2} \\
& \quad \times\left[\exp -\left(\lambda \varepsilon^{* * *}\left(d^{2}(x, y) /\left(2\left(t_{a}-t_{1}\right)\right)\right)\right)\right] \\
& \quad \times \int_{T_{x^{M}}} \exp -\left(\lambda \varepsilon^{* * *}\left|Z^{\prime}\right|^{2} / 2\right) d Z^{\prime} .
\end{aligned}
$$

Therefore, we have (7.15).

Define a operator $S^{(j)}(\lambda ; t)$ by

$$
\begin{aligned}
& S^{(j)}(\lambda ; t)=\boldsymbol{H}\left(\lambda ;\left(A_{j}+B_{j+1}\right) t / N,\left(A_{j}+B_{j}\right) t / N\right) R^{(j)}\left(\lambda ; A_{\jmath}, B_{j} t\right) \cdots \\
& \quad \cdots \boldsymbol{H}\left(\lambda ;\left(A_{1}+B_{1}\right) t / N,\left(A_{1}+B_{1}\right) t / N\right) R^{(1)}\left(\lambda ; A_{1}, B_{1} t\right) \boldsymbol{H}\left(\lambda ; B_{1} t / N, 0\right)
\end{aligned}
$$

and we denote by $S^{(j)}(\lambda ; t ; x, y)$ the kernel function of $S^{(j)}(\lambda ; t)$. Using (7.13) and doing the similar computations as above, we have

$$
\begin{aligned}
\mid S^{(j)} & \left.(\lambda ; t ; x, y)\right|_{(x, y)} \\
\leqq & M_{0}^{2 A} \gamma_{2}^{2 A_{j}}\left[\exp \left(\gamma_{3}(t / N) B_{j}\right)\right](t / N)^{3 A_{j} / 2}\left(\left(A_{j}+B_{j}\right) t / N\right)^{-m / 2} \\
\quad & \times\left[\exp -\left(\lambda \varepsilon^{(4)}\left(d^{2}(x, y) /\left(2\left(A_{j}+B_{j}\right) t / N\right)\right)\right)\right]
\end{aligned}
$$

where $\varepsilon^{(4)}=1-4 \varepsilon$.

Proof of Theorem 1.3. Combining (7.11) and (7.19), we get 


$$
\begin{aligned}
& \sum_{\left(\alpha_{1}, \cdots, \alpha_{k} ; \beta_{1}, \cdots, \beta_{k+1} \in \mathscr{P}\right.}\left|\mathcal{I}\left(\alpha_{1}, \cdots, \alpha_{k} ; \beta_{1}, \cdots, \beta_{k+1} ; x, y\right)\right|_{(x, y)} \\
& \left.\quad \leqq M_{0}^{2 A_{k}+1} \gamma_{2}^{2 A_{k}+1}\left[\exp \left(\gamma_{3} t\right)\right](t / N)^{3 A_{k} / 2} t^{-m / 2}\left[\exp -\left(\lambda \varepsilon^{(4)} d^{2}(x, y) / 2 t\right)\right)\right] \\
& \leqq M_{0} \gamma_{2} t^{-m / 2}\left[\exp \left(\gamma_{3} t\right)\right]\left(\left(1+M_{0} \gamma_{2}(t / N)^{3 / 2}\right)^{N}-1\right)\left[\exp -\left(\lambda \varepsilon^{(4)} d^{2}(x, y) / 2 t\right)\right] \\
& \leqq M_{0} \gamma_{2}\left[\exp \left(\gamma_{3} t\right)\right] t^{-m / 2}(t / N)^{3 / 2}\left[\exp \left(M_{0} \gamma_{3}(t / N)^{1 / 2}\right)\right]\left[\exp -\left(\lambda \varepsilon^{(4)} d^{2}(x, y) / 2 t\right)\right]
\end{aligned}
$$

where $I(\cdots ; \cdots ; x, y)$ is the kernel function of $(7.11)$ and $\varepsilon^{(4)}=1-4 \varepsilon$. So, Theorem 1.3 is obtained.

Remark. The above computation can be slightly moved for general subdivision of $[0, t]$ (Cf. [11]).

Appendix. Growth of the higher order derivatives of $\rho(x, y)$ and $P(x, y)$.

In this appendix, we shall show the growth estimates of $\rho(x, y)$ and $P(x, y)$, defined by $\S 2$, under the assumptions (A.0)-(A.2). First, we give the estimate for $\rho(x, y)$. Namely, we have

Proposition A.1. Assume that (A.0)-(A.1) holds. Then, there exists a positive constant $k_{4}$ such that

$$
\left|\nabla_{y}^{\alpha} \rho(x, y)\right|_{y} \leqq k_{4}\left(\exp k_{4} r\right), \quad r=d(x, y), \quad 0 \leqq|\alpha| \leqq 3,
$$

for any $x, y \in M$.

To show the above proposition, we prepare some lemmas. Remark that the exponential mapping is a diffeomorphism from $T_{x} M$ onto $M$ by the assumptions. Thus, we can introduce the normal coordinate around $x$ (Cf. [13] for the precise notation). By the identification $T_{x} M \cong S_{x} M \times \boldsymbol{R}^{+}$, we shall use the normal polar coordinate $(r, \omega)$, where $\omega=\left(\omega^{2}, \cdots, \omega^{m}\right)$ in a local coordinate of $S_{x} M=$ $\left\{\boldsymbol{\omega} \in T_{x} M ;|\omega|_{x}=1\right\}$ and $r \in \boldsymbol{R}^{+}$. Choosing an orthonormal vectors $e_{2}(\omega), \cdots, e_{m}(\omega)$, at a point $(r, \omega)$, which are perpendicular to radial axis, we may assume that $\left\{e_{2}(\omega), \cdots, e_{m}(\omega)\right\}$ depends smoothly on $\omega$ locally. We put, for $a=2, \cdots, m$,

$$
K_{a}\left(\tau, \omega_{1}\right)=\operatorname{Exp}_{x} \tau\left(\omega+\left(\varepsilon_{1} / r\right) e_{a}(\omega)\right),
$$

for sufficiently small $\varepsilon_{1}$. Since (2) is a geodesic variation, $\left(\partial / \partial \varepsilon_{1}\right) K_{a}$ is a Jacobi field along the curve $K_{a}\left(\tau, \omega_{1}\right)$ for each fixed $\varepsilon_{1}$. Therefore, we can apply the comparison theorem and we have for some constant $k_{41}>0$,

$$
\left\{\begin{array}{l}
\left|\left(\partial / \partial \varepsilon_{1}\right) K_{a}(r, 0)\right|_{y} \leqq k_{41}\left(\exp k_{41} r\right), \\
\left|(\delta / \delta \tau)\left(\partial / \partial \varepsilon_{1}\right) K_{a}(r, 0)\right|_{y} \leqq k_{41}\left(\exp k_{41} r\right),
\end{array}\right.
$$

where $r=d(x, y)$ and $\operatorname{Exp}_{x} r \omega=y$.

Let us use the idices $A, B, C, \cdots=1,2, \cdots, m$ and $a, b, c, \cdots=2,3, \cdots, m$. Denote by $g_{A B}$ the component of the Riemannian metric $g$ with respect to the coordinate $(r, \omega)$, i.e. 


$$
\begin{gathered}
\left\{\begin{array}{l}
g_{11}(r, \omega)=g\left(\left(d E x p_{x}\right)_{r \omega} \omega,\left(d E x p_{x}\right)_{r \omega} \omega\right)=1, \\
g_{1 a}(r, \omega)=g\left(\left(d E x p_{x}\right)_{r \omega} \omega,\left(d E x p_{x}\right)_{r} e_{a}(\omega)\right)=0,
\end{array}\right. \\
g_{a b}(r, \omega)=g\left(\left(d E x p_{x}\right)_{r \omega} e_{a}(\omega),\left(d E x p_{x}\right)_{r \omega} e_{b}(\omega)\right) .
\end{gathered}
$$

Differentiating (5) directly and noticing that $\left(\partial / \partial \varepsilon_{1}\right) K_{a \mid \varepsilon_{1}=0}=\left(d E x p_{x}\right)_{r \omega} e_{a}(\omega)$, we have for any $2 \leqq a, b \leqq m$, with some constant $k_{42}>0$,

$$
\left\{\begin{array}{l}
\left|g_{a b}(r, \omega)\right| \leqq k_{42}\left(\exp k_{42} r\right) \\
\left|\partial_{r} g_{a b}(r, \omega)\right| \leqq k_{42}\left(\exp k_{42} r\right)
\end{array}\right.
$$

Lemma A.2. Under the same assumptions as in Proposition A.1, there exists $a$ positive constant $k_{43}$ such that for any $2 \leqq a, b \leqq m$,

$$
\left|\partial_{c} g_{a b}(r, \omega)\right| \leqq k_{43}\left(\exp k_{43} r\right) \text {. }
$$

Proof. We take a smooth curve $\omega\left(\varepsilon_{2}\right)$ in $S_{x} M$ for sufficiently small $\varepsilon_{2}$ such that $\left(\partial / \partial \varepsilon_{2}\right) \omega(0)=e_{c}, c=2, \cdots, m$. Consider

$$
K_{a}\left(\tau, \varepsilon_{1}, \varepsilon_{2}\right)=\operatorname{Exp}_{x} \tau\left(\omega\left(\varepsilon_{2}\right)+\left(\varepsilon_{1} / r\right) e_{a}\left(\omega\left(\varepsilon_{2}\right)\right)\right) .
$$

Then, $K_{a}\left(\tau, \varepsilon_{1}, \varepsilon_{2}\right)$ is also geodesic variation in two parameters $\varepsilon_{1}, \varepsilon_{2}$ and has the following initial conditions

$$
\left(\partial / \partial \varepsilon_{1}\right) K_{a}\left(0, \varepsilon_{1}, \varepsilon_{2}\right)=0, \quad\left(\partial / \partial \varepsilon_{2}\right) K_{a}\left(0, \varepsilon_{1}, \varepsilon_{2}\right)=0,
$$

and

$$
\left\{\begin{array}{l}
(\delta / \delta \tau)\left(\partial / \partial \varepsilon_{1}\right) K_{a}\left(0, \varepsilon_{1}, \varepsilon_{2}\right)=(1 / r) e_{a}\left(\omega\left(\varepsilon_{2}\right)\right) \\
(\delta / \delta \tau)\left(\partial / \partial \varepsilon_{2}\right) K_{a}\left(0, \varepsilon_{1}, \varepsilon_{2}\right)=\omega^{\prime}\left(\varepsilon_{2}\right)+\left(\varepsilon_{1} / r\right)\left(d / d \varepsilon_{2}\right) e_{a}\left(\omega\left(\varepsilon_{2}\right)\right)
\end{array}\right.
$$

By differentiating the Jacobi equation with respect to $\varepsilon_{1}$ and $\varepsilon_{2}$, and putting $\varepsilon_{1}=\varepsilon_{2}=0$, we get

$$
\begin{aligned}
& \left(\delta^{2} / \delta \varepsilon_{i} \delta \varepsilon_{j}\right)\left(\delta^{2} / \delta \tau^{2}\right) K_{a}(\tau, 0,0)+R\left(\dot{\gamma}(\tau),\left(\delta^{2} / \delta \varepsilon_{i} \delta \varepsilon_{j}\right) K_{a}(\tau, 0,0)\right) \dot{\gamma}(\tau) \\
& \quad=F_{a, \imath, j}(\tau),
\end{aligned}
$$

$i, \jmath=1,2, \gamma(\tau)=\operatorname{Exp}_{x} \tau \omega$, where $F_{a, \imath, j}(\tau)$ is the function of $R, \nabla R,\left(\partial / \partial \varepsilon_{i}\right) K_{a}$, $(\delta / \delta \tau)\left(\partial / \partial \varepsilon_{\imath}\right) K_{a}$ and $(/)\left(/{ }_{j}\right) K_{a}$. Also, we have the following setimate by $(2.3)$ and (2.5),

$$
\left|F_{a, \imath, j}(r)\right|_{\gamma(r)} \leqq k_{44}\left(\exp k_{44} r\right), \quad \gamma(r)=\operatorname{Exp}{ }_{x} r \omega,
$$

with some constant $k_{44}>0$. Therefore, we get by variation of constant

$$
\left|\left(\delta^{2} / \delta \varepsilon_{i} \delta \varepsilon_{j}\right) K_{a}(r, 0,0)\right|_{\gamma(r)} \leqq k_{45}\left(\exp k_{45} r\right),
$$

$\gamma(r)=\operatorname{Exp} p_{x} r \omega$, with some positive constant $k_{45}>0$. Then, in accordance of 
$\omega=\omega^{a} e_{a}, \omega=\left(\omega^{2}, \cdots, \omega^{m}\right)$ as the coordinate of $\omega$, using (13), we have

we get (7).

$$
\begin{aligned}
\partial_{c} g_{a b}(r, \omega)= & \left.\left(d / d \varepsilon_{2}\right) g_{a b}\left(r, \omega\left(\varepsilon_{2}\right)\right)\right|_{\varepsilon_{2}=0} \\
= & g\left(\left(\delta^{2} / \delta \varepsilon_{2} \delta \varepsilon_{1}\right) K_{a}(r, 0,0),\left(\partial / \partial \varepsilon_{1}\right) K_{b}(r, 0,0)\right)_{r(r)} \\
& +g\left(\left(\partial / \partial \varepsilon_{1}\right) K_{a}(r, 0,0),\left(\delta^{2} / \delta \varepsilon_{2} \delta \varepsilon_{1}\right) K_{b}(r, 0,0)\right)_{r(r)},
\end{aligned}
$$

By Lemma A.2 and the definitions of the Christoffel symbols and $\rho(x, y)$, we have

Lemma A.3. Under the same assumptions as in Proposition A.1, there exists a positive constant $k_{46}$ such that the following estimates hold:

$$
\begin{aligned}
& \left|g^{A B}(r, \omega)\right| \leqq k_{46}\left(\exp k_{46} r\right), \\
& \left|\Gamma_{B C}^{A}(r, \omega)\right| \leqq k_{46}\left(\exp k_{46} r\right), \quad r=d(x, y)
\end{aligned}
$$

where $g^{A B}(r, \omega)$ and $\Gamma_{B C}^{A}(r, \omega)$ are the inverse matrix of $g=\left(g_{A B}(r, \omega)\right)$ and the Christoffel symbol of $g$ with respect to the coordinate $(r, \omega)$ respectively. Moreover, we have

$$
\left|\nabla_{y} \rho(x, y)\right|_{y} \leqq k_{46}\left(\exp k_{46} r\right), \quad r=k(x, y) .
$$

Now, let $\omega^{2}, \cdots, \omega^{m}$ be the coordinates on part of $S_{x} M$. We denote by $D^{p, v}$ the differential operator, $v=\left(v_{2}, \cdots, v_{m}\right)$,

$$
D^{p, v}=(\partial / \partial r)^{p}\left(\partial / \partial \omega^{2}\right)^{v_{2}} \cdots\left(\partial / \partial \omega^{m}\right)^{v_{m}} .
$$

Differentiating the Jacobi equation successively and using the variation of constant, we get the following, which gives Proposition A.1, because of the definition of $\rho(x, y)$ (Cf. [3]).

Lemma A.4. Under the same assumptions as in Proposition A.1, there exists a positive constant $k_{47}$ such that

$$
\left|D^{p, v} J_{a}(r, \omega)\right|_{\gamma(r)} \leqq k_{47}\left(\exp k_{47} r\right), \quad a=2, \cdots, m,
$$

$\gamma(r)=\operatorname{Exp}{ }_{x} r \omega$, where $J_{a}(\tau, \omega)=\left(\partial / \partial \varepsilon_{1}\right) K_{a}(\tau, 0)$.

Remark. (i) Bérard [1] has a similar estimate for $\rho(x, y)$ when the case that $M$ is a universal coverting space of a compact manifold.

(ii) Assuming the boundedness of higher order derivatives of the curvature tensor, we get the more higher order growth estimate for $\rho(x, y)$.

Next, we give the higher order estimate for $P(x, y)$. Namely, we get

Proposition A.5. Under the assumptions (A.0)-(A.2), there exists a positive constant $k_{48}$ such that for any $0 \leqq|\alpha| \leqq 3$, 


$$
\begin{aligned}
& \left|\left(D_{x}\right)^{\alpha} P(x, y)\right|_{(x, y)} \leqq k_{48}\left(\exp k_{48} r\right), \\
& \left|\left(D_{y}\right)^{\alpha} P(x, y)\right|_{(x, y) \leqq} k_{48}\left(\exp k_{48} r\right), \quad r=d(x, y) .
\end{aligned}
$$

Remark. If we obtain the above proposition, we have Proposition 2.6, because the adjoint operator $D^{*}$ can be written by using $D$.

Before proving Proposition A.5 generally, we first observe the following:

Lemma A.6. Under the same assumptions as in Proposition A.5, there exists a positive constant $k_{49}$ such that

$$
\left\{\begin{array}{l}
\left|D_{x} P(x, y)\right|_{(x, y)} \leqq k_{49}\left(\exp k_{49} r\right), \\
\left|D_{y} P(x, y)\right|_{(x, y)} \leqq k_{49}\left(\exp k_{49} r\right), \quad r=d(x, y) .
\end{array}\right.
$$

Proof. Let $\left\{e_{1}(y), \cdots, e_{m}(y)\right\}$ be an orthonormal basis of $T_{y} M$ and put $\xi_{\imath}(x, y)=P(x, y) e_{i}(y)$. Take $\left\{f_{1}(x), \cdots, f_{m}(x)\right\}$ as an orthonormal basis of $T_{x} M$ also. Let $\eta_{j}\left(\varepsilon_{1}\right)$ be a smooth curve such that $\eta_{j}(0)=x$ and $\left(d / d \varepsilon_{1}\right) \eta_{j}(0)=f_{j}(x)$, $j=1, \cdots, m$. Then, we get

$$
D_{f_{j}(x)} \xi(x, y)=\left(\delta / \delta \varepsilon_{1}\right) \xi_{j}\left(\eta_{j}\left(\varepsilon_{1}\right), y\right)_{1}=0 .
$$

Consider the variation $K_{j}\left(\tau, \varepsilon_{1}\right)=\operatorname{Exp}_{y} \tau\left(\omega+\left(\varepsilon_{1} / r\right) f_{j}(y)\right)$, where $\operatorname{Exp}_{y} r \omega=x$ and $f_{j}(y)$ is the parallel transport along the geodesic from $x$ to $y$, i.e. $f_{j}(y)=$ $P(y, x) f_{j}(x)$. Also, we define $\xi_{j}\left(\tau, \varepsilon_{1}\right) \in E_{K_{j}\left(\tau, \varepsilon_{1}\right)}, j=1, \cdots, m$ by

$$
(\delta / \delta \tau) \xi_{j}\left(\tau, \varepsilon_{1}\right)=0, \quad \xi_{j}\left(0, \varepsilon_{1}\right)=e_{j}(y),
$$

for each field $\varepsilon_{1}$. Differentiating covariantly (2.15) with respect to $\varepsilon_{1}$, we get

$$
(\delta / \delta \tau)\left(\delta / \delta \varepsilon_{1}\right) \xi_{j}\left(\tau, \varepsilon_{1}\right)+\Omega\left((\delta / \delta \tau) K_{\jmath},\left(\delta / \delta \varepsilon_{1}\right) \xi_{j}\right)=0
$$

where $\Omega$ denotes the curvature tensor of $D$. Since $\left(\delta / \delta \varepsilon_{1}\right) K_{j \mid \varepsilon_{1}=0}$ is a Jacobi field along $\gamma(\tau)=E x p_{x} r \omega$, we get by Lemma 2.4

$$
\begin{aligned}
(d / d \tau)\left|\left(\delta / \delta \varepsilon_{1}\right) \xi_{j}(\tau, 0)\right| \\
\quad \leqq 2|\Omega|_{\gamma(\tau)}\left|(\delta / \delta \tau) K_{j}(\tau, 0)\right|_{\gamma(\tau)}\left|\left(\delta / \delta \varepsilon_{1}\right) K_{j}(\tau, 0)\right|_{\gamma(\tau)}\left|\xi_{j}\right|_{\gamma(\tau)} \\
\quad \leqq 2 k_{49}^{\prime}\left(\exp k_{49}^{\prime} r\right)
\end{aligned}
$$

with some constant $k_{49}^{\prime}>0$. Thus, we have

$$
\begin{aligned}
\left|D_{x} P(x, y)\right|_{(x, y)}^{2} & =\sum_{i, \jmath=1}^{m}\left|D_{f_{j}(x)} \xi_{i}(x, y)\right|_{x}^{2} \\
& \leqq 2 k_{49}\left(\exp k_{49} r\right)
\end{aligned}
$$

which proves the first inequality of (20). The second one is obvious by using $P(x, y) P(y, x)=I d$. 
Proof of Proposition A.5 is easily obtained by differentiating (2.15) covariantly and doing the similar computations as in the proof of Lemma A.6. Thus, we get the desired results.

\section{REFERENCES}

[1] BERARD, P., On the wave equation on a Riemannian manifold without conjugate points, Math. A., 155 (1977), 249-276.

[2] BERGER, M., Le spectre des variétés riemannianes, Revue roumaine Math. Phys. et appl., t.13 (1968), 915-931.

[3] Berger, M., Gauduchon, P. And Mazet, E., Le spectre d'une variété Riemannienne, Lecture notes in Math., 140, Springer, 1971.

[4] Cheeger, J. AND Ebin, D.G., Comparison theorems in Riemannian geometry, Amsterdam, North-Holland, 1975.

[5] ChenG, S.Y., LI, P. AND YAU, S. T., On the upper estimate of the heat kernel of complete Riemannian manifolds, Amer. J. Math., 103 (1981), 1021-1063.

[6] Chorin, A.J., Hughes, J.R., McCracken, M.F. And Marseden, J.E., Product formulas and numerical algorithms, Commun. Pure Appl. Math., 31 (1978), 205-379.

[7] DEWITT, B.S., Dynamical theory in curved spaces I, A review of the classical and quantum action principles, Rev. Mod. Phys., 29 (1957), 377-379.

[8] Feynman, R.P., Space time approach to non-relativistic quantum mechanics, Rev. Mod. Phys., 20 (1948), 367-387.

[9] Friedman, A., Partial differential equations of parabolic type, Pretice-Hall, 1964.

[10] Fujiwara, D., A construction of the fundamental solution for the Schrödinger equation, J. d'Anal. Math., 35 (1979), 41-96.

[11] Fujiwara, D., Remarks on convergence of the Feynman path integrals, Duke Math. J., 47 (1980), 559-600.

[12] InOUE, A. AND MAEDA, Y., On integral transformations associated with a certain Riemannian metric, Proc. of Japan Acad., 58 (1982), 281-283.

[13] InOUE, A. AND MAEDA, Y., On integral transformations associated with a certain Lagrangian, as a prototype of quantization, J. Math Soc. Japan 37 (1985), 129-244.

[14] Lichnerowicz, A., Global theory of connection and holonomy groups, Noordholl International Publ., 1976.

[15] Molchanov, S. A., Diffusion processes and Riemannian geometry, Russian Math. Surveys, 30 (1) (1975), 1-63.

[16] VAN VLECK, J.H., The correspondence principle in statistical interpretation of quantum mechanics, Proc. Nat. Acad. Sci., 14 (1928), 431-455.

Department of Mathematics

Faculty of Science and Technology

KeIO UNIVERSITY

YOKOHAMA, 223, JAPAN 